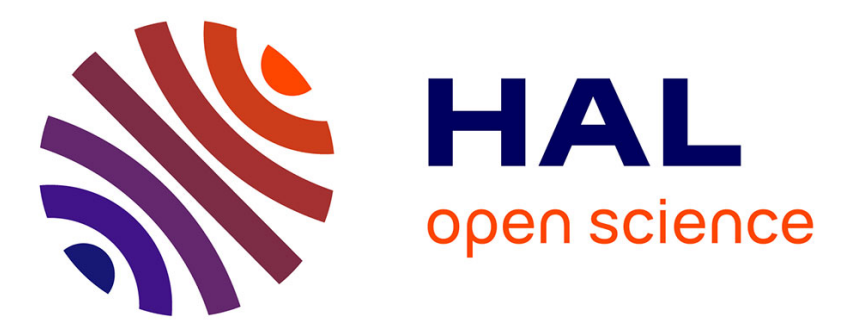

\title{
Bayesian inversion of joint SH seismic and seismoelectric data to infer glacier system properties
}

Franco Macchioli-grande, Fabio Zyserman, Leonardo B. Monachesi, Laurence Jouniaux, Marina Rosas-carbajal

\section{- To cite this version:}

Franco Macchioli-grande, Fabio Zyserman, Leonardo B. Monachesi, Laurence Jouniaux, Marina Rosascarbajal. Bayesian inversion of joint SH seismic and seismoelectric data to infer glacier system properties. Geophysical Prospecting, 2020, 68, pp.1633 - 1656. 10.1111/1365-2478.12940 . hal-03079400

\section{HAL Id: hal-03079400 https://hal.science/hal-03079400}

Submitted on 5 Nov 2021

HAL is a multi-disciplinary open access archive for the deposit and dissemination of scientific research documents, whether they are published or not. The documents may come from teaching and research institutions in France or abroad, or from public or private research centers.
L'archive ouverte pluridisciplinaire HAL, est destinée au dépôt et à la diffusion de documents scientifiques de niveau recherche, publiés ou non, émanant des établissements d'enseignement et de recherche français ou étrangers, des laboratoires publics ou privés. 


\section{Geophysical Prospecting}

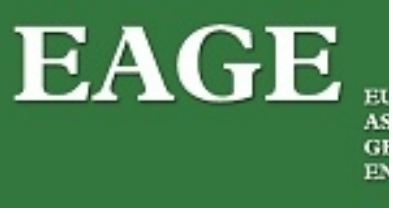

\section{Bayesian inversion of joint SH seismic and seismoelectric data to infer glacier system properties}

\begin{tabular}{|r|l|}
\hline Journal: & Geophysical Prospecting \\
\hline Manuscript ID & Draft \\
\hline Manuscript Type: & Original Manuscripts \\
\hline Author: & n/a \\
\hline Complete List of Authors: & $\begin{array}{l}\text { Macchioli-Grande, Franco; Universidad Nacional de La Plata Facultad de } \\
\text { Ciencias Astronómicas y Geofísicas, Geofísica Aplicada } \\
\text { Zyserman, Fabio; Universidad Nacional de La Plata Facultad de Ciencias } \\
\text { Astronómicas y Geofísicas, Geofísica Aplicada } \\
\text { Monachesi, Leonardo; Universidad Nacional de Río Negro, Instituto de } \\
\text { Investigación en Paleobiología y Geología } \\
\text { Jouniaux, Laurence; CNRS- IPGS, Institut de Physique du Globe de } \\
\text { Strasbourg } \\
\text { Rosas-Carbajal, Marina; Institut de Physique du Globe de Paris, } \\
\text { Systhèmes volcaniques }\end{array}$ \\
\hline Keyword: & Electromagnetic, Inversion, Seismic \\
\hline &
\end{tabular}

\section{SCHOLARONE \\ Manuscripts}




\title{
Bayesian inversion of joint $\mathrm{SH}$ seismic and seismoelectric data to infer glacier system properties
}

\author{
Franco Macchioli-Grande ${ }^{1 *}$ | Fabio Zyserman $\mathrm{PhD}^{1 *}$ | \\ Leonardo Monachesi $\mathrm{PhD}^{2 *}$ | Laurence Jouniaux $\mathrm{PhD}^{3 *}$ \\ Marina Rosas-Carbajal $\mathrm{PhD}^{4 *}$
}

${ }^{1}$ CONICET, Facultad de Ciencias Astronómicas y Geofísicas, Universidad Nacional de La Plata, La Plata, Buenos Aires, 1900, Argentina

${ }^{2}$ CONICET, Instituto de Investigación en Paleobiología y Geología, Universidad Nacional de Río Negro, General Roca, Río Negro, 8332, Argentina

${ }^{3}$ Université de Strasbourg et CNRS, Institut de Physique du Globe de Strasbourg UMR 7516, Strasbourg, Grand Est, 67084, France

${ }^{4}$ Université de Paris, Institut de Physique du Globe de Paris, CNRS, UMR 7154, Paris, Île-de-France, 75005, Paris

\section{Correspondence}

Franco Macchioli-Grande, Facultad de Ciencias Astronómicas y Geofísicas, Universidad Nacional de La Plata, La Plata, Buenos Aires, 1900, Argentina Email: fmacchio@fcaglp.unlp.edu.ar

Funding information

FZ acknowledges support from CONICET through grant PIP 112-201501-00192. LJ aknowledges support from CNRS INSU through the PICS program. MRC acknowledges support from the AXA Research Fund.
In glacial studies, properties such as glacier thickness and the basement permeability and porosity are key to understand the hydrological and mechanical behaviour of the system. The seismoelectric method is a geophysical tool with a huge potential in determining key properties of glacial environments. Here we analytically model the generation of seismic and seismoelectric signals by means of a shear horizontal ( $\mathrm{SH}$ ) seismic wave source on top of a glacier overlying a porous basement. Considering a one dimensional setting, we compute the seismic waves and the electrokinetically induced electric field. We then analyze the sensitivity of the seismic and electromagnetic data to relevant model parameters, namely depth of the glacier bottom, porosity, permeability, shear modulus and saturating water salinity of the glacier basement. Furthermore, we study the possibility of inferring these key parameters from a set of synthetic data, adopting a Bayesian framework to pay particular attention to the uncertainty of the model parameters mentioned above. We tackle the resolution of the probabilistic inverse problem with two strategies: 1) we compute

\footnotetext{
*Equally contributing authors.
} 
the marginal posterior distributions of each model parameter solving multidimensional integrals numerically and 2) we use a Markov chain Monte Carlo (MCMC) algorithm to retrieve a collection of model parameters that follows the posterior probability density function (pdf). Both methodologies are able to obtain the marginal distributions of the parameters and estimate their mean and standard deviation. The MCMC algorithm performs better in terms of numerical stability and amount of iterations needed to characterize the distributions. The inversion of seismic data alone is not able to constrain the values of porosity and permeability further than the prior distribution. In turn, the inversion of the electric data alone, and the joint inversion of seismic and electric data are useful to constrain these parameters as well as other glacial system properties. Furthermore, the joint inversion reduces the uncertainty of the model parameters estimates and provides more accurate results.

\section{KEYWORDS}

Electromagnetics, Inversion, Modelling, Parameter Estimation, Seismics

\section{1 | INTRODUCTION}

Characterizing glacier system properties has been a matter of interest in the field of electric and electromagnetic prospecting methods throughout the last two decades. The spontaneous potential (SP) method consists in the measurement of the steady state and natural potentials that are present on the near surface (Fournier, 1989). Kulessa and Murray (2003); Kulessa et al. (2006a) were able to characterize hydraulic properties of subglacial sediments, as long as hydraulic processes within them using this method and Kulessa et al. (2003); French et al. (2006) employed SP to study the impact of earth tides in the subglacial hydrological system. In this context, the seismoelectric method appears as a potential utility in glacial studies to detect the interface between the glacier and the basement and to determine hydrogeophysical properties of the subglacier system.

Seismoelectrics is a lively research realm in which recent results in theory (Jougnot et al., 2013; Jardani and Revil, 2015; Ren et al., 2016; Grobbe and Slob, 2016; Munch and Zyserman, 2016; Gao et al., 2017a,b; Guan et al., 2017; Zyserman et al., 2017a,b; Guan et al., 2017; Fiorentino et al., 2017; Monachesi et al., 2018a; Dietrich et al., 2018; Gao et al., 2019) as well as laboratory tests (Bordes et al., 2015; Hu et al., 2015; Holzhauer et al., 2016; Peng et al., 2017; Devi et al., 2018) were achieved. Seismoelectromagnetics aims at combining the resolution of the seismic method with the sensitivity of the electric methods to fluid content in porous rocks. Both sesimoelectric and electromagnetic signals originate from the electrokinetic phenomenon. The seismoelectric method uses a seismic source to create the relative motion between the fluid and the rock matrix. This relative motion induces an equivalent density current leading to 
an electromagnetic field accompanying the seismic wave propagation. Moreover, strong seismoelectric signals can arise at interfaces of media with different electrokinetic properties (Haartsen and Pride, 1997; Garambois and Dietrich, 2002). At such interfaces the electric current density is unbalanced, inducing an electric dipole and thus an electric field propagating at the speed of the light in the medium and detected at the surface. The resulting electrical potential distribution can provide important information about the subsurface. A detailed account of results on most topics of interest in seismoelectrics can be found in the review of Jouniaux and Zyserman (2016) and in the book by Revil et al. (2015).

On a first approach, Kulessa et al. (2006b) recorded seismoelectric signals on Glacier de Tsanfleuron, Switzerland, using a vertical sounding geometry. They inferred the electromagnetic waves to be generated by electrokinetic conversion of seismic waves within the snow pack and near the dry-wet ice and ice-bed interfaces. In laboratory tests, Liu et al. (2008) proved that an electric double layer is formed at the boundary between permafrost and unfrozen soil, and observed seismoelectric conversions originated there due to the electric and mechanical contrasts between both media; on the other hand Zyserman et al. (2012) observed electroseismic conversions at the same interface in a numerical study of methane-hydrate reservoirs. Later, Mahardika (2013) was able to produce numerical synthetic recordings compatible with the data collected by Kulessa et al. (2006b), treating the snow-glacial environment in a similar way as he did with the vadose zone-aquifer zone in an unsaturated porous medium (Revil and Mahardika, 2013). Monachesi et al. (2018b) developed analytic expressions for the coseismic and interface signals produced in a glacial environment when a SH seismic wave traverses it. They observed that the electric interface response originated at the glacier bottom, which proved to be up to three orders of magnitude stronger than the coseismic signal, is proportional to the electric current density at this depth, and depends on textural and electrical properties of the basement. Quite recently, Siegert et al. (2018) interpreted, from seismoelectric soundings of the West Greenland Ice Sheet, arrival times from the till layer beneath the ice-sheet base fully compatible with previous data obtained with seismic amplitude variation with offset (AVO) surveys. This study firmly heartens future developments for the hydrological and mechanical characterization of ice-sheet substrates by means of the seismoelectric method.

The existing literature on inversion of seismoelectric data is not, to our knowledge, very broad. Jardani et al. (2010) employed a finite element algorithm to model the seismoelectric response over a two dimensional stratified medium hosting a reservoir partially saturated with oil. They used this algorithm to generate synthetic seismic and seismoelectric data and performed a stochastic joint inversion procedure using an Adaptive Metropolis algorithm. This allowed them to obtain the posterior probability density functions (posterior pdf) of the parameters (see Section 5 for a review of these concepts) of the different geological units of the model, comprising permeability, porosity, electrical conductivity, bulk modulus of the dry porous frame, bulk modulus of the pore fluid, bulk modulus of the solid phase and shear modulus of the formations. They were able to recover the permeability within one order of magnitude, electrical conductivity and other material properties, with the exception of the porosity, which was not well constrained by the inversion. Although the obtained pdfs are mostly centered around the true values, they show large dispersion, even in logarithmic scale. More recently, Mahardika et al. (2012); Mahardika (2013), employing a similar approach, performed an inversion of synthetic data corresponding to the occurrence of a fracking event in a two-layered system. The authors concluded that when using noiseless data, its electric portion contains more information than the seismic one, and that the model parameters obtained via joint inversion are more accurate than the ones obtained by inverting the seismic time-series alone. It is worth to mention also the works of Guan et al. (2013); Chen and Yang (2013) and Bonnetier et al. (2019), which deal with an electrolyte-saturated homogeneous poroelastic medium. Proposing a deterministic inversion methodology, Guan et al. (2013) recovered the permeability of different fluid saturated homogeneous porous formations from seismoelectric well logs, deriving the amplitude ratio of electric signal to acoustic pressure (REP) of Stoneley waves, and obtaining the permeability from an expression involving the tangent of its phase. They determined 
the conditions under which the electric conductivity and the electrokinetic coupling coefficient can be recovered from a two-step inversion. On the other hand, Chen and Yang (2013) and Bonnetier et al. (2019) carried out a stability analysis of the inverse problem for the possible recovery of the same two parameters from knowledge of the seismoelectric fields, but based on theoretical studies of electroseismic conversions stated by Pride (1994).

In the present paper we propose a Bayesian framework to analyze the information that can be recovered about relevant model parameters of a glacial environment. Assuming a one dimensional geometry, we simulate the noiseless data following the analytic expressions for the electric field and seismic waves obtained by Monachesi et al. (2018b). The seismoelectric theoretical framework used in the forward modeling is the one presented by Pride (1994), broadly used in seismoelectric studies (Garambois and Dietrich, 2002; Hu et al., 2007; Haines et al., 2007a,b; Hu and Gao, 2011; Zyserman et al., 2012; Guan et al., 2013; Warden et al., 2012, 2013; Kröger et al., 2014; Bordes et al., 2015; Zyserman et al., 2015; Gao et al., 2017a; Guan et al., 2017; Zyserman et al., 2017a). The probabilistic inversion of synthetic data is tackled using two different approaches to estimate the distribution of relevant model parameters and their uncertainties. On the one hand, we calculate the posterior pdf of each model parameter computing multidimensional integrals. We numerically compute the latter by means of the Cuba library (Hahn, 2005), which offers a variety of Monte Carlo and deterministic methods. On the other hand, we solve the probabilistic inverse problem using a Markov chain Monte Carlo algorithm: the DiffeRential Evolution Adaptive Metropolis (DREAM) (Vrugt et al., 2009). This is a highly optimized algorithm that combines the capability of MCMC methods of ensuring convergence to the target distribution (Tarantola, 2005) with features of Differential Evolution algorithms (Sambridge and Drijkoningen, 1992; Sen and Stoffa, 1992) which allow a proper scale and orientation of the proposal distribution employed to sample the posterior pdf. This approach results in a collection of model parameters that follow the posterior pdf. Histograms of the marginal distributions and the uncertainty estimates can be computed from such collection.

The paper is structured as follows: Section 2 comprises the formulation of the equations for the seismoelectric problem in a glacial environment. Section 3 details the model parameters we are interested in studying, and Section 4 explains how the synthetic data are computed. Section 5 presents the principles of Bayesian inference applied to our problem, and the methodology used to compute the posterior marginal probabilities and the full posterior pdf distribution using a Markov chain Monte Carlo scheme. In Section 6 we present the main results in terms of a sensitivity analysis and the Bayesian inference with the aforementioned techniques. Finally, Section 7 outlines the main findings and conclusions.

\section{2 | FORWARD MODEL}

Following Monachesi et al. (2018b) we assume a one-dimensional medium constituted by a single layer on top of a half-space in contact at a given depth denoted by $z_{\mathrm{b}}$ (see Fig.1). The top layer represents the ice body of the glacier, and is assumed to be an elastic medium, while the half-space represents the glacier basement, which we assume to be a porous medium fully-saturated with water. The seismic source of the system, located at the glacier surface is a shearing force, parallel to the $x$ axis acting on a horizontal infinite plane. Under these assumptions the source can only induce displacements in the $x$ direction, with amplitudes depending only on depth.

The mechanical equation that governs the wave propagation in the glacier, written in the space-frequency domain, assuming an $e^{i \omega t}$ time dependence, is given by (Aki and Richards, 2002):

$$
-\omega^{2} \rho_{\text {ice }} u_{x}-G_{\text {ice }} \frac{\partial^{2} u_{x}}{\partial z^{2}}=F^{s}(\omega) \delta(z)
$$


where $\omega$ is the angular frequency, $\rho_{\text {ice }}$ and $G_{\text {ice }}$ stand for the mass density and the shear modulus of the ice composing the glacier, and $u_{x}$ is the displacement. The right hand side of eq. (1) represents the shearing source acting on the surface $(z=0) ; F^{s}(\omega)$ is the shearing force per unit area and $\delta(z)$ is the Dirac delta function.

In order to model the seismoelectric response, we assume that the net electric charge is zero and the magnetic permeability is the one of the vacuum. With these assumptions, the electric and magnetic fields in the glacier will satisfy the following simplified form of Maxwell's equations:

$$
\begin{aligned}
-\sigma_{\text {ice }} E_{x}-\frac{\partial H_{y}}{\partial z} & =0, \\
\frac{\partial E_{x}}{\partial z}+i \omega \mu_{0} H_{y} & =0,
\end{aligned}
$$

where $E_{x}$ and $H_{y}$ are the electric and magnetic fields, respectively, $\sigma_{\text {ice }}$ is the electric conductivity of the glacier and $\mu_{0}=4 \pi \times 10^{-7} \mathrm{~N} \mathrm{~A}^{-2}$ is the vacuum magnetic permeability. Note that the displacement currents are not accounted for in eqs. (2) and (3); this is the common assumption for shallow seismoelectric surveys (Hu and Liu, 2002; Haines and Pride, 2006; Bordes et al., 2015; Gao et al., 2017a; Guan et al., 2017). This form of Maxwell's equations is commonly known as magneto quasi-static approximation (MQS). It has been analyzed within the frame of seismoelectrics in the last two mentioned references, and previously by other authors (Løseth et al., 2006; Rapetti and Rousseaux, 2014) in more general contexts.

To model the seismoelectric response in the basement we use the equations derived by Pride (1994). If the electroosmotic feedback is neglected in Biot's equations, as it is usually assumed for frequencies that vary from $10 \mathrm{~Hz}$ to $1 \mathrm{kHz}$, range of interest for shallow seismoelectric surveys (tens to hundreds of meters) (Hu and Liu, 2002; Haines and Pride, 2006; Warden et al., 2013; Bordes et al., 2015; Guan et al., 2017; Gao et al., 2017a), Pride's equations can be written as follows:

$$
\begin{aligned}
& -\omega^{2} \rho_{\mathrm{b}} u_{s, x}-\omega^{2} \rho_{w} u_{f, x}-G_{\mathrm{b}} \frac{\partial^{2} u_{s, x}}{\partial z^{2}}=0, \\
& -\omega^{2} \rho_{w} u_{s, x}-\omega^{2} g_{0} u_{f, x}+i \omega \frac{\eta_{\mathrm{w}}}{\kappa} u_{f, x}=0, \\
& -\sigma_{\mathrm{b}} E_{x}-\frac{\partial H_{y}}{\partial z}=i \omega \frac{\eta_{\mathrm{w}}}{\kappa} L_{0} u_{f, x}=j_{v}, \\
& \frac{\partial E_{x}}{\partial z}+i \omega \mu_{0} H_{y}=0 .
\end{aligned}
$$

In these equations, $u_{s, x}$ and $u_{f, x}$ are the average solid and relative fluid displacements respectively, $\rho_{\mathrm{b}}$ is the bulk mass density, which can be computed as $\rho_{\mathrm{b}}=\rho_{\mathrm{S}}(1-\phi)+\phi \rho_{\mathrm{W}}$, being $\rho_{\mathrm{S}}$ the mass density of the solid grains constituting the basement porous matrix, $\rho_{\mathrm{w}}$ the mass density of water and $\phi$ the porosity of the medium. $G_{\mathrm{b}}$ is the basement rock matrix shear modulus, $g_{0}$ the Biot's low frequency inertial coupling coefficient, computed as $g_{0}=F \rho_{\mathrm{W}}$ (Santos et al., 2004, 2005; Zyserman et al., 2012), where $F$ is the formation factor given by $F=\phi^{-\hat{m}}$, being $\hat{m}$ the cementation exponent, $\eta_{\mathrm{w}}$ is the water viscosity and $\kappa$ the permeability of the porous rock matrix. The right hand side in eq. (6) is the electric current density, source of the electromagnetic signals, and can be referred to as the viscous current density $j_{v}$, whereas $\sigma_{\mathrm{b}} E_{x}$ is the conduction current, being $\sigma_{\mathrm{b}}$ the electric conductivity of the basement. The coefficient $L_{0}$ is the electrokinetic coupling (Pride, 1994); it creates, in this model, the coupling between the seismic wave and the electric and magnetic fields. If this coupling is zero, there are no seismo-electromagnetic conversions. Within the 
seismic frequency band it can be written as (Pride, 1994; Warden et al., 2013; Bordes et al., 2015):

$$
L_{0}=-\frac{\epsilon_{\mathrm{w}} \zeta}{\eta_{\mathrm{w}} F}\left(1-2 \frac{d}{\Lambda}\right)
$$

where $\zeta$ is the zeta potential and $\epsilon_{\mathrm{W}}$ is the permittivity of water, $d$ the Debye length and $\Lambda$ a geometrical parameter, related to the matrix pore structure.

In order to solve the problem stated by eqs. (1)-(7) it is necessary to establish boundary conditions for the displacements and the electromagnetic fields, both in the boundaries of the system $(z=0$ and $z \rightarrow \infty)$ and at the interface between both media $\left(z=z_{\mathrm{b}}\right)$. Once the boundary conditions are stated, the problem can be analytically solved taking advantage of the decoupling of the mechanical and electromagnetic equations; we first solve the mechanical problem, and then, the obtained solution is used to derive the final solutions for the electric and magnetic fields. Their final expressions are given in the Appendix; the full derivation is detailed in (Monachesi et al., 2018b).

\section{3 | MODEL PARAMETERS}

We first present the subsurface model parameters that we are interested in characterizing and those we assume to be known in advance. Then we describe the rock physics models published in the literature which we use to relate our unknown model parameters to others needed to solve the forward problem. In the previous section we established that both the glacier and the basement are homogeneous. As in a geophysical survey we have direct access to the ice, we consider known -and constant- all its relevant parameters (see values in Table 1). The ice shear modulus is computed as $G_{\text {ice }}=v_{\text {ice }}^{2} \rho_{\text {ice }}$.

Therefore, we consider the basement parameters listed in Table 1 as the free ones (the ones to be determined by an inversion procedure are in boldface). Other parameters, independent of the listed ones but considered to remain constant, are the water viscosity $\eta_{\mathrm{w}}$ and the water electric permittivity $\varepsilon_{\mathrm{w}}$. The water viscosity is not expected to change significantly from the chosen value (see Table 1) due to the studied geological setting whereas the electric permittivity could in principle be considered as a possible free model parameter. However, as it can be seen in Gueguen and Palciauskas (1994, Fig. IX.7), for temperatures near and above $0^{\circ} \mathrm{C}, \varepsilon_{\mathrm{w}}$ remains almost constant. Consequently, we considered $\varepsilon_{\mathrm{W}}$ to be constant, and used $\varepsilon_{\mathrm{W}}=85 \varepsilon_{0}$ (Gueguen and Palciauskas, 1994) in this work.

We turn now the attention to the non free parameters of the model. For the basement electric conductivity $\sigma_{\mathrm{b}}$, we used Archie's law (Archie, 1942; Mavko et al., 2009)

$$
\sigma_{\mathrm{b}}=\frac{\sigma_{\mathrm{w}}}{F}
$$

where $\sigma_{\mathrm{w}}$ is the electric conductivity of the saturating water in the glacier basement, and $F=\phi^{-\hat{m}}$ is the formation factor, which expresses the reduction of the water conductivity due to the presence of the electrically isolating rock matrix. As described in Monachesi et al. (2018b), for water containing just dissolved $\mathrm{NaCl}$, we can use $\sigma_{\mathrm{w}}=\sum_{l=N a^{+}, \mathrm{Cl}^{-}}\left(e z_{l}\right)^{2} b_{l} N_{l}$, where $e=1.6 \times 10^{-19} \mathrm{C}$ is the electron electric charge, and $z_{l}$ is the ions' valence; we used $z_{l}=1$ for both species. The ions' mobility $b_{l}$ and concentration $N_{l}$, both dependent on the salinity $C_{0}$, are calculated following Carcione et al. (2003). Notice that the way in which we compute the bulk electric conductivity implies that the basement rock matrix is assumed to be clean, i.e., it does not contain a significant amount of clay. Had we considered otherwise, we would have used a corrected version, including the surface conductivity (Schön, 1996), for which several models exist (a couple of them are discussed in Zyserman et al. (2017b)). 
To finish with, we analyze the electrokinetic coupling coefficient $L_{0}$ in eq.(8), which depends on the non free parameters $\zeta, d$ and $\wedge$. We follow Pride (1994) and Santos (2009) for the treatment of their dependence on the free model parameters. For the $\zeta$ potential we use

$$
\zeta=\left(0.008+0.26 \log _{10}\left(C_{0}\right)\right)\left(\frac{p H-5}{2}\right)
$$

In this work $p H=7$ is employed. The parameter $d$, as we mentioned above, is the Debye length, computed as

$$
d^{-1}=\sqrt{\sum_{l=N a^{+}, C l^{-}} \frac{N_{l}\left(e z_{l}\right)^{2}}{\varepsilon_{\mathrm{W}} k T}} .
$$

Here $k=1.3807 \times 10^{-23} \mathrm{~J} / \mathrm{K}$ is the Boltzmann's constant and $T$, the temperature; we use for $z_{l}$ and $N_{l}$ the same values as for the water conductivity $\sigma_{\mathrm{b}}$. Finally, the parameter $\Lambda$, which can be seen as a pore surface to volume ratio (Johnson et al., 1987; Pride, 1994) is computed as

$$
\Lambda=2 \sqrt{F \kappa}
$$

\section{4 | COMPUTATION OF THE SYNTHETIC DATA}

The values of the model parameters used to compute the synthetic data are listed in Table 1 . Those not shown in the table can be obtained from them using the formulas given or referenced above. Of course, at this stage we also need to choose values for the free parameters, i.e. the ones we use in next sections in the inversion study. The chosen values are referred to as the "true values" and they are displayed between round brackets. Notice that when computing the synthetic data we decided instead of assigning arbitrary values to the basement permeability $k$ and shear modulus $G_{\mathrm{b}}$, to use a rock physics model to obtain them. For the estimation of the basement solid matrix shear modulus $G_{\mathrm{b}}$ we use Walton's model (Mavko et al., 2009), appropriate to model unconsolidated media (Pride, 2005; Bordes et al., 2015; Dupuy et al., 2016):

$$
G_{\mathrm{b}}=\frac{1}{10}\left[\frac{3(1-\phi)^{2} \hat{C}^{2} P}{\pi B^{2}}\right] \text {, with } B=\frac{1}{4 \pi}\left(\frac{1}{G_{s}}+\frac{1}{G_{s}+\lambda_{c}}\right)
$$

In this equation, $\hat{C}$ is the coordination number, related to the packing of the spheres building the solid aggregate, $P$ is the hydrostatic pressure and $\lambda_{c}$ is Lamé's coefficient of the effective grain material, and is computed as $\lambda_{c}=K_{s}-\frac{2}{3} G_{s}$, where $K_{s}$ is the bulk modulus of the solid grains. In this work we consider $\hat{C}=9$. The hydrostatic pressure can be computed as $P=P_{0}+\rho_{\text {ice }} g z_{\mathrm{b}}$, being $P_{0}=101325 \mathrm{~Pa}$ the air pressure at the surface of the Earth and $g=9.81 \mathrm{~m} \mathrm{~s}^{-2}$ the gravity of Earth. Taking $G_{s}=45 \mathrm{GPa}$ and $K_{s}=36 \mathrm{GPa}$, representative for the shear and bulk moduli of quartz grains, respectively (Mavko et al., 2009), we obtain, as displayed in Table 1, $G_{\mathrm{b}}=0.477 \mathrm{GPa}$. To compute the value for the basement permeability to be employed in the computation of the synthetic field data we use (Mavko et al., 2009)

$$
\kappa=\frac{1}{72}\left(\frac{D_{q}}{\phi F}\right)^{2} \frac{\phi^{3}}{(1-\phi)^{2}}
$$

in this model, referred to as Kozeny-Carman equation, $D_{q}=8 \times 10^{-5} \mathrm{~m}$ is the diameter of the quartz grains. Using $\phi=0.3$, one obtains $\kappa=2.11 \times 10^{-12} \mathrm{~m}^{2}$, as displayed in Table 1 . 
Having all the model parameters set, we can turn our attention to the $\mathrm{SH}$ seismic source and the simulation of noisy data. To calculate the time signature of the source $F^{s}(t)$, we use a Ricker wavelet with peak frequency $f_{\text {peak }}=120 \mathrm{~Hz}$; its peak amplitude, located at $t=8 \times 10^{-3} \mathrm{~s}$, is set so that the maximum amplitude of the force per unit area at the surface is $1 \mathrm{~N} \mathrm{~m}^{-2}$. Monachesi et al. (2018b) followed previous studies of shear wave sources (Krawczyk et al., 2013; Bordes, 2005) to compute a source amplitude close to actual field values. In that case the aim was to test the feasibility of the seismoelectric method by estimating the minimum amplitude that would be recorded at the surface, for which a realistic amplitude value is crucial. In the present study we focus on testing the feasibility of retrieving the model parameters from the data. We set a recording time of $1.024 \mathrm{~s}$, and Fourier transform the source using a sampling period of 0.5 $\times 10^{-3} \mathrm{~s}$ to take $N_{t}=2048$ samples in the frequency domain. The seismic and electromagnetic responses are computed in the frequency domain, and inverse Fourier transformed to recover them back in the time domain (see Appendix). The number of samples is chosen as a power of 2 in order to efficiently employ the Fast Fourier Transform (FFT) when going to the frequency domain and its inverse to move backwards to the time domain. In Fig. 2 we show the time signature of the source, and the computed time traces for solid displacement and electric field at the surface. We set $z=0$ and for each frequency, we use eq.(31) to get $u_{x}(0)$ and eq. (36) to get $E_{x}(0)$. Afterwards, we inverse Fourier transform them and obtain the traces displayed in the figure. We do not employ the magnetic field in the inversion because, as stated in Monachesi et al. (2018b), its amplitude at the surface is very small compared to its amplitude below the glacier basement, and thus very difficult to be measured.

The synthetic data $\mathbf{d}=\left(\mathbf{d}_{E}, \mathbf{d}_{S}\right)$ are computed according to the following expressions

$$
\begin{aligned}
& d_{E, i}=\left(1+p_{E} r_{i}\right) \hat{d}_{E, i}, \\
& d_{S, i}=\hat{d}_{S, i}+p_{S} r_{i} .
\end{aligned}
$$

In this equations, $\hat{d}_{E, i}$ is the $i$-th component of the vector containing the $N_{t}$ time samples of the noise-free computed electric field and $\hat{d}_{S, i}$ the corresponding component for the noise-free computed solid displacements. $r_{i}$ is the $i$-th component of a $N_{t}$-dimension random noise vector following a zero mean normal distribution, while $p_{E}$ and $p_{S}$ are positive factors controlling the amplitude of the noise we want to add to the noiseless electric and seismic signals, respectively. Note that the noise added to the electric and seismic noise-free data, $n_{E, i}$ and $n_{S, i}$, are different. For the electric case we assume $n_{E, i}=p_{E} r_{i} \hat{d}_{E, i}$, i.e, the noise is proportional to the signal so its amplitude is controlled by $\hat{d}_{E, i}$ and $p_{E}$, while for the seismic case $n_{S, i}=p_{S} r_{i}$, i.e, the noise is independent from the signal (Chen et al., 2007). One way to quantify the noise level in the seismic case is by means of the signal-to-noise ratio (SNR) (Robinson and Treitel, 2000) which can be defined as the ratio of the squared $\mathrm{I}_{2}$ - norms of the clean data and the noise, i.e,

$$
\mathrm{SNR}=\frac{\left\|\hat{\mathbf{d}_{S}}\right\|_{2}^{2}}{\left\|\mathbf{n}_{S}\right\|_{2}^{2}}=\frac{\left\|\hat{\mathbf{d}_{S}}\right\|_{2}^{2}}{p_{S}^{2}\|\hat{\mathbf{r}}\|_{2}^{2}},
$$

which means that $p_{S}$ can be chosen to yield a predefined value for SNR. The noise term corresponding to both electric and seismic signals can be thought as the error of the measurements simulated by the synthetic data set. Given the way these are calculated, the errors result to be uncorrelated.

\section{5 | BAYESIAN INFERENCE OF RELEVANT MODEL PARAMETERS}

The resolution of the inverse problem aims at determining the model parameters presented before, together with their uncertainties. Following a probabilistic approach implies that, instead of searching an exact model, we intend to 
characterize the full posterior pdf of the model parameters. This function is related to the previous knowledge about the model, and to the pdf of the data set given a certain model through Bayes' theorem:

$$
p(\mathbf{m} \mid \mathbf{d})=\frac{p(\mathbf{m}) p(\mathbf{d} \mid \mathbf{m})}{p(\mathbf{d})},
$$

where $\mathrm{p}(\mathbf{m} \mid \mathbf{d})$ is the posterior pdf of the model parameters conditioned on the data set; $p(\mathbf{m})$ is the prior pdf, which accounts for the a priori information of the model parameters; $p(\mathbf{d} \mid \mathbf{m})$ is the probability of the data conditioned on the model parameters, which also appears in the literature as the likelihood function; and $p(\mathbf{d})$ is the pdf of the data, also known as evidence, which is constant for a fixed model parametrization. Since the present work involves such type of parametrization, we can dismiss the evidence and, instead of using the equality in eq. (18), we employ a proportional relationship to obtain the posterior pdf:

$$
p(\mathbf{m} \mid \mathbf{d}) \propto p(\mathbf{m}) p(\mathbf{d} \mid \mathbf{m})
$$

\section{1 | Marginal distributions via numerical integration}

As a first approach to characterize the uncertainty of the model parameters $m$ in a Bayesian framework, we attempt to calculate their marginal posterior distributions. Following Brandt (1989), let $f(m)$ be the joint probability density of $M$ variables $\mathbf{m}=\left(m_{1}, m_{2}, \ldots, m_{M}\right)$. The marginal distribution of a single parameter $m_{r}$ is given by:

$$
g_{r}\left(m_{r}\right)=\int_{-\infty}^{\infty} \int_{-\infty}^{\infty} \ldots \int_{-\infty}^{\infty} f(\mathbf{m}) d m_{1} d m_{2} \cdots d m_{r-1} d m_{r+1} \cdots d m_{M}
$$

and it can be interpreted as the probability density of $m_{r}$, accounting for the uncertainty of the remaining model parameters. Then the marginal distribution $g_{r}\left(m_{r}\right)$ can be used to compute the mean $\mu_{r}$ and variance $\sigma_{r}^{2}$ of $m_{r}$ as

$$
\mu_{r}=\int_{-\infty}^{\infty} m_{r} g_{r}\left(m_{r}\right) d m_{r}, \quad \sigma_{r}^{2}=\int_{-\infty}^{\infty}\left(m_{r}-\mu_{r}\right)^{2} g_{r}\left(m_{r}\right) d m_{r} .
$$

In our case the vector of model parameters, which refers to the glacier depth and basement properties, is characterized as follows:

$$
\mathbf{m}=\left(\phi, z_{\mathrm{b}}, \log _{10}\left(G_{\mathrm{b}}\right), \log _{10}(\kappa), \log _{10}\left(C_{0}\right)\right)
$$

Instead of working with the proper values for the bulk modulus, the permeability and the salinity, the inversion and the previous sensitivity analysis are performed in $\log _{10}$ scale. This is because these parameters present order-of-magnitude variations in nature, and so it is more reasonable to explore the space of model parameters using a $\log _{10}$ scale (Tarantola, 2005).

We assume a Gaussian behaviour of the data errors and uniform prior distributions for the model parameters (i.e. Jeffreys priors for $\log _{10}$ variables, Tarantola (2005)). Thus, $p(\mathbf{m} \mid \mathbf{d})$ has the form:

$$
p(\mathbf{m} \mid \mathbf{d})=V \exp \left(-\frac{\Phi}{2}\right)
$$


where $V$ is a normalization factor and $\Phi$ is the data misfit, whose explicit form will be given in Section 6.2.

In this way, if we consider for example the porosity, its marginal distribution can be obtained according to the eq.(21) as

$$
g_{\phi}(\phi)=\int_{z_{\mathrm{b}, \min }}^{z_{\mathrm{b}, \max }} \int_{G_{\mathrm{b}, \min }}^{G_{\mathrm{b}, \max }} \int_{K_{\min }}^{K_{\max }} \int_{C_{0, \min }}^{C_{0, \max }} p(\mathbf{m} \mid \mathbf{d}) d z_{\mathrm{b}} d G_{\mathrm{b}} d k d C_{0},
$$

where the minimum and maximum values of the integrals result from the non-zero limits of the corresponding prior distributions. Notice that we can compute the coefficient $V$ appearing in eq. (23) by employing the condition that the integration of $p(\mathbf{m} \mid \mathbf{d})$ over the whole parametric space is equal to one. Therefore,

$$
V^{-1}=\int_{\phi_{\min }}^{\phi_{\max }} \int_{z_{\mathrm{b}, \min }}^{z_{\mathrm{b}, \max }} \int_{G_{\mathrm{b}, \min }}^{G_{\mathrm{b}, \max }} \int_{K_{\min }}^{\kappa_{\max }} \int_{C_{0, \min }}^{C_{0, \max }} \exp \left(-\frac{\Phi}{2}\right) d z_{\mathrm{b}} d G_{\mathrm{b}} d k d C_{0} d \phi,
$$

and the mean value and variance are given by

$$
\mu_{\phi}=V^{-1} \int_{\phi_{\min }}^{\phi_{\max }} \phi g_{\phi}(\phi) d \phi, \quad \sigma_{\phi}^{2}=V^{-1} \int_{\phi_{\min }}^{\phi_{\max }}\left(\phi-\mu_{\phi}\right)^{2} g_{\phi}(\phi) d \phi .
$$

The mean values and variances of the other model parameters are obtained in the same way.

To accomplish this task, we resort to multidimensional numerical integration, which is not easy to implement (Press et al., 2007). A straightforward generalization of one dimensional numerical integration leads to very long computing times even for integrals in low dimensions as the ones we are dealing with. We therefore employ the multidimensional integration library Cuba (Hahn, 2005), which offers four different approximation methods to compute the integrals. We tested all of them, and because of its better performance we decided to work with the Divonne routine.

\section{2 | MCMC inversion}

Analytical solutions to eq. (18) are seldom possible. MCMC algorithms can be used to sample the posterior pdf by searching through the parameter space (Tarantola and Valette, 1982; Mosegaard and Tarantola, 1995; Sambdridge and Mosegaard, 2002; Ter Braak, 2006; Vrugt et al., 2009; Rosas-Carbajal et al., 2015). Monte Carlo methods draw samples of the desired distribution and Markov chains guide properly this sampling in an efficient manner. Under certain conditions, Markov chains become independent from the initial state and, after a burn in period, they converge to its stationary distribution (Gilks et al., 1995). Therefore, we have to construct a Markov chain such that its stationary distribution is the one we are seeking. There are many ways to accomplish this task, but in general the MetropolisHastings algorithm is used. Briefly, given the current state of the chain $\mathbf{m}_{t}$, a candidate point $\mathbf{m}^{*}$ is drawn from a proposal distribution which then is accepted with probability $\alpha$ :

$$
\alpha\left(\mathbf{m}_{\mathbf{t}} \mid \mathbf{m}^{*}\right)=\min \left\{1, \frac{\pi\left(\mathbf{m}^{*}\right) q\left(\mathbf{m}_{\mathbf{t}} \mid \mathbf{m}^{*}\right)}{\pi\left(\mathbf{m}_{\mathbf{t}}\right) q\left(\mathbf{m}^{*} \mid \mathbf{m}_{\mathbf{t}}\right)}\right\}
$$


where $\pi(\cdot)$ is the target distribution and $q(\cdot)$ is the proposal distribution. If the candidate point is accepted, then $\mathbf{m}_{t+1}=$ $\mathbf{m}^{*}$. Otherwise, the chain remains in the current state (Gilks et al., 1995). We employ an Adaptive Metropolis scheme, in which the proposal distribution is updated according to the samples that are continuously drawn from the posterior (Hassan et al., 2009). This scheme, after a certain amount of iterations, will produce samples that follow the posterior distribution we are looking for. Since we are using uniform priors, the acceptance rule can be calculated in terms of the ratio of the likelihoods of two states (Mosegaard and Tarantola, 1995).

The MCMC algorithm is implemented through the DiffeRential Evolution Adaptive Metropolis (DREAM) algorithm (Vrugt et al., 2009), which efficiently copes with non-linear problems. A remarkable feature of this algorithm is that it uses several Markov chains in parallel to converge to the target distribution. The information recovered by the chains is mixed using a formulation that incorporates properties of genetic algorithms. The following equation describes such behaviour:

$$
\mathbf{z}^{i}=\mathbf{x}_{t-1}^{i}+\gamma\left(\mathbf{X}_{t-1}^{r_{j}}-\mathbf{X}_{t-1}^{r_{2}}\right)+\epsilon, \quad r_{1} \neq r_{2} \neq i
$$

where $\mathbf{z}^{i}$ is a sample of the $\mathrm{i}$-th chain, which combines a sample of the previous iteration of the same chain $\mathbf{x}_{t-1}^{i}$ with a linear combination of members of the rest of the chains $\mathbf{X}_{t-1}^{r_{1}}$. The constant $\gamma$ sets the jump rate with which the chains sample different regions of the parameter space. This allows to automatically tune the scale and orientation of the proposal distribution. $\epsilon$ is the perturbation of ergodicity, which controls how much a state of the chain depends on the previous one. The choice of the different values follows the criterion stated in Vrugt et al. (2009), where the optimal value is given by the expression $\gamma=2.38 / \sqrt{2 \delta}$, where $\delta$ is the dimensionality of the problem, which refers to the number of parameters desired to be constrained in the inversion. This quotient comes from the properties of Random Walk Metropolis and this factor is appropriate for Gaussian distributions. Finally, the convergence of the chains to the target distribution is expected to be reached when the Gelman-Rubin factor $(\hat{R})$ is less than 1.2 (Gelman et al., 1992).

\section{6 | RESULTS}

We start by briefly describing the synthetic seismic and electric signals produced or electrokinetically induced respectively by the seismic source. We follow by studying the sensitivity of the data to the model parameters and we end this section by analyzing the outcome of the chosen inversion methods.

\section{1 | Forward model: Seismic and electric data}

The time signature of the noiseless solid displacement computed at the surface (Fig. 2) exhibits four events evenly spaced in time; three of them are clearly observable in the figure and the last one is a tiny hump. The first signal arrives at the very moment the source energy is released, because it corresponds to the direct wave. A second arrival occurs 0.22 $\mathrm{s}$ after the detonation of the source. This is the time needed by the wave in its way forth and back between the surface and the glacier-basement interface (the velocity of the seismic wave is $1800 \mathrm{~m} / \mathrm{s}$ and $z_{\mathrm{b}}=200 \mathrm{~m}$ ), corresponding to a first reflection at the interface. The third event arrives at twice the time of the second one, corresponding to a second reflection at the glacier-basement interface. Finally, the fourth arrival is recorded at a time $0.66 \mathrm{~s}$, and corresponds to a third reflection. Note that the amplitudes of the consecutive events are diminishing with time. This is because when the wave hits the interface, part of its energy is released to the basement as a transmitted wave, while the remaining energy travels back to the surface as a reflected wave. The amplitudes of both reflected and transmitted waves at the $n$-th 
incidence at $z=z_{\mathrm{b}}$ are given by eqs. (10) and (12), respectively.

Turning now the attention to the electric field (Fig. 2(c)), it can be noticed that three events are recorded within the considered time window. The first one arrives at $0.11 \mathrm{~s}$, which is half the arrival time of the second seismic event. This recorded arrival is due to an interface response (IR) produced when the seismic wave hits the interface. As it was studied in Monachesi et al. (2018b) the source of this IR is the jump in the electric current density occurring at the interface between the glacier and its basement (the current density is zero at the ice because of the absence of fluids, and is different from zero at the glacier basement). Once the seismic wave hits the interface, the produced IR travels to the surface at a speed given by $\sqrt{2 \omega /\left(\mu_{0} \sigma\right)}$; this is approximately $10^{5} \mathrm{~m} / \mathrm{s}$ at the source peak frequency, much higher than that of the seismic wave. This explains why the observed arrival time for the first event is half the time of the first seismic arrival. Regarding the second and third events, it is now clear from simple inspection of Fig. 2 that they respectively correspond to IR's produced by the second and third incidences of the seismic wave over the interface. Note that as in the case of the solid displacement, the electric field shows an amplitude decay for the consecutive events. This is because the amplitude of the electric field is proportional to the amplitude of the electric current density (see eqs. (36) and (40)) which in turn is proportional to the amplitude of the solid displacement.

\section{2 | Sensitivity analysis in terms of data misfits}

To evaluate the possibility of retrieving the relevant model parameters through data inversion, we study their influence on the seismic and electric data misfits. We choose an $I_{2}$-norm measure for the electric field and solid displacements data misfits, which is adequate for Gaussian errors (Rosas-Carbajal et al., 2014, 2015). The seismic and electric misfits are:

$$
\Phi_{E}=\sum_{i=1}^{N_{t}}\left(\frac{d_{E, i}-\hat{d}_{E, i}}{p_{E} d_{E, i}}\right)^{2}, \quad \Phi_{S}=\sum_{i=1}^{N_{t}}\left(\frac{d_{S, i}-\hat{d}_{S, i}}{p_{S}}\right)^{2},
$$

respectively. The denominators in $\Phi_{E}\left(\Phi_{s}\right)$ are their corresponding standard deviations of the $i$-th electric field (solid displacements) errors. The misfit for the joint problem is

$$
\Phi=\Phi_{E}+\Phi_{S}
$$

which is replaced in eq. 23 to calculate $p(m \mid d)$. To study the sensitivity of each relevant parameter, we calculate the misfit between the synthetic data and the forward model response computed using the true model parameters, except for the parameter chosen to perform the analysis.

First of all, we evaluate how many events are appropriate to be considered in the sensitivity analysis and consequently in the inversion study. It is clear that $n$ cannot be higher than 3 , because the third event has already a very small amplitude (Fig. 2(b)). Moreover, the seismic and electric traces with $n=1$ have already all the physical information we want to recover. However, increasing the number of events provides a larger data set, which from a statistical point of view reinforces the probabilistic approach, according to the law of large numbers (Jeffreys, 1998). This is why, we choose to use the traces with three events, which leads to a data set of $N=2 N_{t}=4096$.

We now determine through a misfit analysis whether the seismic and electric data are sensitive or not to changes in the model parameters. We perform this study by varying each one of them at a time over a predefined prior domain, while leaving the others fixed and equal to the true values. The best scenario is that the log-misfits exhibit a clear and sharp minimum around the true value for each parameter. For the electric data, we employed three percentages of 
error to corrupt the true model response: $5 \%, 10 \%$ and $15 \%$. In the seismic case, we generated synthetic data sets using SNR=10, 20 and 50. The electric misfit exhibits a sharp minimum near the true value for the porosity parameter, whereas the seismic data misfits present smooth minima near the true value of porosity for the three SNR cases (Fig. 3). Both the electric and seismic misfit show a sharp minimum near the true value of the depth (Fig. 4), though the curves differ considerably from the behavior as a function of the porosity, presenting an oscillatory behaviour. The electric and seismic misfits also present sharp minima as a function of the shear modulus in all cases (Fig. 5). The seismic case minima are a little bit smoother than the electric ones, but are still very distinctive. The electric misfit show sharp minima near the true value of the permeability, while the seismic misfit remain constant for permeabilities lower than $10^{-10} \mathrm{~m}^{2}$ (Fig. 6). This fact is correlated with the results of the inversion of seismic data (Section 6.4). Finally, the misfit analysis of the salinity is only referred to the electric data because the physics of the problem do not relate the seismic response to this parameter. There are also sharp minima near the true value of the concentration (Fig. 7).

We can conclude that the synthetic data are sensitive to the whole parameter set. In general, the misfits become larger for the lowest percentage of error in the electric data and the highest SNR in the seismic data (both cases represent the less realistic synthetics). This fact is key for the election of the synthetic set for the inversion, since the numerical computation of the marginal distributions have problems dealing with large misfits. We select the electric data with an error of $10 \%$ of the amplitude of the response of the true model and the seismic data with an SNR=20..

\section{3 | Multidimensional numerical integration}

We first attempt to obtain the posterior marginal distribution of each model parameter by integration, and from them the resulting means and standard deviations. The computation of the marginal pdfs (see eq. (24) for the porosity case) is a numerically challenging task, even with a dedicated software as the one we employ.

Due to the large values of the misfit $\Phi$, the computation has to be performed in quadruple precision in order to avoid the integrand to be identically zero. Indeed, as the minimum misfit value is $\Phi=4096$, the highest value the integrand of the marginal pdfs can take is $\exp (-2048)$, i.e., approximately $3.7 \times 10^{-890}$, a much lower value than the inferior limit the double precision allows to represent. Furthermore, a simple inspection of Fig. 3 to Fig. 7 shows that the misfit takes values higher than $10^{4}$ but for a small interval enclosing the true values. We mention this because beyond this value the integrand is taken as zero, even in quadruple precision. Thus, in order to be able to compute the marginal pdfs, i.e., the numerical algorithm perform an appropriate sampling near the true values, we were forced to restrict the integration limits to rather small intervals around them, which is equivalent to considering small prior pdf ranges for $p(m)$.

Having clarified this point, we show in Fig. 8 the obtained posterior marginal pdfs of the model parameters. They were obtained following a procedure that we here describe for the porosity, but is similar for the whole parameter set. The calculations follow this scheme: we choose 20 porosity values equally distributed among the prior limits. We compute the integral given in eq. (24), i.e. a four dimensional integral, using the Divonne routine from the Cuba library. Afterwards, we estimate the reciprocal of the normalization factor $V$, by simply applying a one dimensional Simpson's rule using the obtained discrete marginal distribution values as ordinates, and the chosen values as abscissae. This result is used to normalize the set of calculated $g_{\phi}\left(\phi_{i}\right), i=1, \ldots, 20$, which we display in Fig. 8(a). Finally, the mean value $\mu_{\phi}$ and the variance $\sigma_{\phi}^{2}$ (see eq.(26)) are in turn computed by again applying Simpson's rule in one dimension. In Table 2 we display the mean values and standard deviations obtained for all the model parameters. The results obtained by this methodology are, we deem, deceptively promising. The marginal distributions are very narrow and their peaks are very close to the respective true values, which is reflected in the computed standard deviations. This is particularly true for $z_{b}$, for which just one value of $g_{z_{b}}\left(z_{b, i}\right), i=1, \ldots, 20$ is not zero. However, these results are at least a consequence of having reduced the integration limits to small intervals around the true values. We consider that this 
fact alone is enough to explore another methodology to characterize the model parameters uncertainty. In addition, the computational cost of the discussed numerical integration method is not low, because to compute each marginal at least $10^{6}$ forward problem computations are needed.

\subsection{Inversion via the DREAM algorithm}

We now evaluate the possibility of retrieving the full posterior pdf of the model parameters using the DREAM algorithm. We perform the inversion of both seismic and electric data sets separately and jointly. For all cases, we use three parallel Markov chains, such that the model proposals of each chain are created accounting for the current state of the two others (see eq. 28). The outcome of this process is a set of models that follow the posterior pdf. To represent this pdf, we display histograms of the last $50 \%$ of the chains states, once convergence has been achieved. For all cases, we employed uniform priors: $[0.15,0.45]$ for $\phi,[150,250]$ for $z_{b}$, [6,9] for $\log _{10}\left(G_{b}\right),[-14,-9]$ for $\log _{10}(\kappa)$ and $[-4,-1]$ for $\log _{10}\left(C_{0}\right)$. The last parameter is only considered in the inversion of the electric and the seismoelectric data, as referred in Section 6.2.

We first present the results concerning the inversion of seismic data alone. Figs. 9(a)-(d) display how the chains sample the parameter space (to clarify the curves, we only display a single chain but the behaviour is similar to the remaining two chains). The expected result is that the process of sampling eventually converges near the true value of each parameter. We tested different values of $\gamma$, around the optimal value of 0.85 (corresponding to $\delta=4$, the number of parameters involved) in order to improve the exploration of the parameter space. In the case of $\phi$, the chains sample the complete model space defined by the prior distribution without converging to a smaller uncertainty range (Fig. 9 (a)), despite the relatively high number of iterations employed ( $8 \times 10^{5}$ iterations). A straightforward convergence of the chains near the true value can be observed for $z_{b}$ (Fig. 9(b)), and the path of the chains for $G_{b}$ has a fairly similar behaviour (Fig. 9 (c)), except that the chains oscillate around the true value in the end, but with a small amplitude. Finally, the behaviour of $k$ is similar to that of $\phi$ (Fig. 9(d)), that is, the inversion of seismic data is not able to constrain this value further than the uniform prior pdf established. Besides collecting the sets of model parameters accepted, the algorithm stores the value of $p(\mathbf{m} \mid \mathbf{d})$ of each accepted set of parameters (see eq.23). This value is expected to be approximately $-N_{t} / 2$ (-1024 in this case) for models that reproduce statistically the data inverted. The chains indeed move around this value (Fig. 9(e)).

We evaluate the results of the inversion through histograms of the marginal distributions of the model parameters (Fig. 10). These histograms are built using the last 50\% of the chains shown in Fig. 9, that is, only after the chains are considered to have converged according to the criteria by Gelman \& Rubin (Gelman et al., 1992). In this way, histograms are representative of the marginal posterior pdf of each parameter, accounting for the uncertainty of all the other parameters considered (see Eq 20). $z_{b}$ and $G_{b}$ are the best resolved parameters of this inversion (Figs. 10(b) and (c) respectively). Their histograms are rather bell-shaped, with peaks near the true model values. Unlike the previously mentioned parameters, $\phi$ and $\kappa$ are not well resolved (Figs. 10(a) and (d) respectively). This is evident from their respective histograms, nearly flat and with maxima far from the true values. In conclusion, the only reliable parameters in this inversion case are $z_{b}$ and $G_{b}$.

The inversion of the electric data led to significantly improved results. In this case, the optimal value of $\gamma$ is 0.75 (corresponding to $\delta=5$ ), but the inversion worked with $\gamma=2$, using $8 \times 10^{5}$ iterations as in the seismic case. Since the results of the inversion of the electric data set do not differ significantly from the joint case, we only display the histograms of the model parameters. These parameters were all constrained in this inversion case (Figs. 12(a)-(e)). All of them are fairly bell-shaped and their maxima are very close to the true values. In Table 2 we compare the parameters' estimates values and uncertainties to the true values used to build the synthetic data. Here it can be clearly observed how the inversion of electric data alone performs better that the corresponding of seismic data alone. Besides being 
able to retrieve one more model parameter, the mean values estimated are closer to the true values, and the uncertainty ranges systematically smaller. For example, the true value of the permeability expressed as $\log 10(\mathrm{k}[\mathrm{m} 2])$ is -11.68 . The inversion of seismic data results in the range of [-12.49,-9.69] of $68 \%$ probability (i.e., one standard deviation), whereas in the electric case the range is $[-11.76,-11.58]$.

Finally, we consider the joint inversion of seismic and electric data. This case required $9 \times 10^{5}$ iterations and a $\gamma$ value of 0.5 , smaller than in the electric case and closer to the optimal value of 0.75 mentioned above. Figs. 11(a)-(e) show the chains searching through the parameter space. In the case of $\phi$, the chains oscillate around the true value with a high amplitude but without covering the entire range defined in the prior distribution (Fig. 11(a)). The chains for $z_{b}$ exhibit, as in the seismic case, an immediate convergence to the true value (Fig. 11(b)). $G_{b}, k$ and $C_{0}$ display tiny oscillations around the true value (Figs. 11(c), (d) and (e)), which implies that the chains managed to constrain the parameter space into a small range within the prior pdf. The values of $p(\mathbf{m} / \mathbf{d})$ end up being close to -2048 , which is half the amount of data points (Fig. 11(f)), as expected.

The histograms that represent the marginal distributions of the model parameters are, as in the electric case inversion, fairly bell-shaped and with maxima almost coincident with the true values (Fig. 13). Taking a look at Table 2 , the mean values of all the model parameters match the true values. Interestingly, adding the seismic data in a joint inversion scheme contributes to further constraining the uncertainty ranges of the model parameters. The uncertainty range of the permeability, following the analysis of the separate inversions, is further reduced in the joint case, reaching the interval $[-11.76,-11.6]$ for the uncertainty ranges of the whole parameters). This result reinforces the advantages of seismoelectric data over seismic data stated in Section 1 (Mahardika et al., 2012; Mahardika, 2013). It is clear that the electric data alone already provide a great improvement in the inversion results, but the joint case is able to slightly improve the determination of the uncertainties of the parameters.

\section{7 | CONCLUSIONS}

We have studied the seismoelectric response to a $\mathrm{SH}$ seismic source deployed on top of a glacier, and by solving the inverse problem with a probabilistic approach, we were able to analyze the feasibility of recovering relevant geophysical and hydrogeophysical information, such as glacier depth, along with porosity, permeability, and bulk shear modulus of the basement, as well as the salinity of the water saturating the rock. We performed a response sensitivity analysis to these relevant parameters by studying the data misfits, and observed that they were good candidates to be employed as free parameters in an inversion study.

The first attempt to perform the inversion was carried out by means of multidimensional integration, which allowed us to obtain marginal distributions of the model parameters. This was successful to a certain extent, because the integration range does not reflect a prior able to properly explore the parameter space. This is related to the value of the misfits, which have at least an order of magnitude of $10^{3}$. The exponential of such numbers is very difficult to calculate even in quadruple precision without obtaining a null result. Another drawback is that obtaining a marginal distribution curve in a very small portion of the prior demands a huge number of computations of the forward model ( $10^{6}$ for each parameter).

Given the limitations encountered with numerical integration, we performed a Bayesian inversion applying an MCMC algorithm. This allowed us to work with the electric and seismic set separately and jointly and to obtain histograms of the marginal distributions of the model parameters in each case. In accordance to what was observed in the sensitivity analysis, the inversions were able to retrieve the relevant model parameters (with exception of the porosity and the permeability in the seismic case). The inversion of electric data alone performed better than that of the 
seismic data alone, and the joint inversion of both data sets resulted in the best estimates of all model parameters, in terms of mean values closer to the true ones and also in uncertainty ranges that were smaller. Each case demanded no more than $9 \times 10^{5}$ iterations (forward computations), which compared with the total of $5 \times 10^{6}$ evaluations of the forward model of the numerical integration makes it a valuable tool to characterize glacier basement properties, with $a$ priori information that does not require a significant closeness to the true values. Therefore, we were able to retrieve the parameters that characterize the basement properties of a glacier environment with Bayesian Monte Carlo inversion, and the uncertainty estimates are reasonable to encourage field work in this direction. This is a promising result that ratifies the importance of combining geophysical methods to study improve the geophysical and hydrogeophysical characterization of glacial areas. 
TAB LE 1 Values of model parameters used in the present study. Those not shown in this table can be obtained from them using the formulas given or referenced in this work. Parameters displayed in boldface are the inversion study free ones, their constant values between round brackets -the referred to as "true values"- are the ones used to compute the noiseless synthetic field data. Notice that, as explained in Section 3, the true values for $k$ and $G_{b}$ are computed in terms of $\phi$ from rock physics models. Values marked with $a^{\star}$ symbol are taken from Collins et al. (2016), those marked with $a^{\ddagger}$ symbol are given in Petrenko and Whitworth (1999). The vacuum permittivity is taken to be $\epsilon_{0}=8.85 \times 10^{-12} \mathrm{~F} \mathrm{~m}^{-1}$.

\begin{tabular}{|c|c|c|c|}
\hline \multicolumn{4}{|c|}{ Model parameters } \\
\hline \multicolumn{4}{|c|}{ Glacier ice } \\
\hline Density, $\rho_{\text {ice }}\left[\mathrm{kg} \mathrm{m}^{-3}\right]$ & $900^{\star}$ & S-wave phase velocity, $v_{\text {ice }}\left[\mathrm{m} \mathrm{s}^{-1}\right]$ & $1800^{\star}$ \\
\hline Electric conductivity, $\sigma_{\text {ice }}\left[\mathrm{S} \mathrm{m}^{-1}\right]$ & $10^{-5}$ & & \\
\hline \multicolumn{4}{|c|}{ Glacier basement } \\
\hline Porosity, $\phi$ & $(0.3)$ & Water mass density, $\rho_{\mathrm{W}}\left[\mathrm{kg} \mathrm{m}^{-3}\right]$ & 1000 \\
\hline Cementation exponent, $\hat{m}$ & 1.35 & Water viscosity, $\eta_{\mathrm{w}}[\mathrm{Pa} \mathrm{s}]$ & $1.7 \times 10^{-3}$ \\
\hline Mass density of the solid grains, $\rho_{\mathrm{s}}\left[\mathrm{kg} \mathrm{m}^{-3}\right]$ & 2600 & Salinity, $C_{0}[\mathrm{Mol} / \mathrm{I}]$ & $\left(5 \times 10^{-3}\right)$ \\
\hline Permeability, $\kappa\left[\mathrm{m}^{2}\right]$ & $\left(2.11 \times 10^{-12}\right)$ & Permitivity of water, $\epsilon_{\mathrm{w}}\left[\mathrm{F} \mathrm{m}^{-1}\right]$ & $85 \epsilon_{0}$ \\
\hline Matrix shear modulus, $G_{\mathrm{b}}[\mathrm{Pa}]$ & $\left(4.77 \times 10^{8}\right)$ & Temperature, $T[\mathrm{~K}]$ & 273 \\
\hline Top depth, $z_{\mathrm{b}}[\mathrm{m}]$ & (200) & & \\
\hline
\end{tabular}


TAB LE 2 Mean value $\mu$ and standard deviation $\sigma$ for the five random variables considered, obtained by means of numerical computation of the integrals in eq. (26), columns three and four, and using the DREAM algorithm, single seismic inversion -columns five and six-, single electromagnetic inversion -columns seven and eight- and joint inversion -columns nine and ten.

\begin{tabular}{lccccc} 
& & Numerical integration & \multicolumn{3}{c}{ DREAM } \\
& & & Seismic inversion & Electric inversion & Joint inversion \\
\hline Variable & True value & $\mu \pm \sigma$ & $\mu \pm \sigma$ & $\mu \pm \sigma$ & $\mu \pm \sigma$ \\
\hline$\phi$ & 0.3 & $0.3 \pm 8 \times 10^{-4}$ & $0.29 \pm 0.093$ & $0.31 \pm 0.046$ & $0.3 \pm 0.038$ \\
\hline$z_{\mathrm{b}}[\mathrm{m}]$ & 200 & $200 \pm 0.0$ & $200 \pm 0.004$ & $200 \pm 0.0001$ & $200 \pm 0.0001$ \\
$\log _{10}\left(G_{\mathrm{b}}[\mathrm{Pa}]\right)$ & 8.68 & $8.7 \pm 3 \times 10^{-39}$ & $8.67 \pm 0.031$ & $8.68 \pm 0.015$ & $8.68 \pm 0.012$ \\
$\log _{10}\left(\kappa\left[\mathrm{m}^{2}\right]\right)$ & -11.68 & $11.78 \pm 5 \times 10^{-12}$ & $-11.09 \pm 1.4$ & $-11.67 \pm 0.09$ & $-11.68 \pm 0.076$ \\
$\log _{10}\left(\mathrm{C}_{0}[\mathrm{Mol} / \mathrm{l}]\right)$ & -2.3 & $-2.29 \pm 2 \times 10^{-10}$ & - & $-2.28 \pm 0.08$ & $-2.295 \pm 0.072$ \\
\hline
\end{tabular}


1

2

3

4

5

6

7

8

9

10

11

12

13

14

15

16

17

18

19

20

21

22

23

24

25

26

27

28

29

30

31

32

33

34

35

36

37

38

39

40

41

42

43

44

45

46

47

48

49

50

51

52

53

54

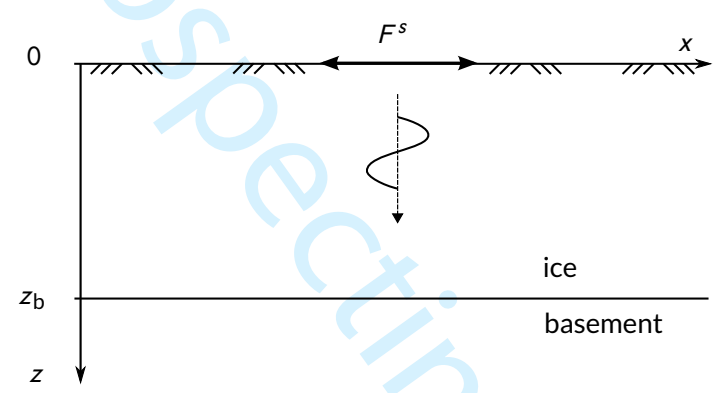

FIGURE 1 Schematic representation of the one-dimensional system considered in this study. The ice body is assumed to be an elastic medium, while the porous basement is treated as a poroelastic medium. 


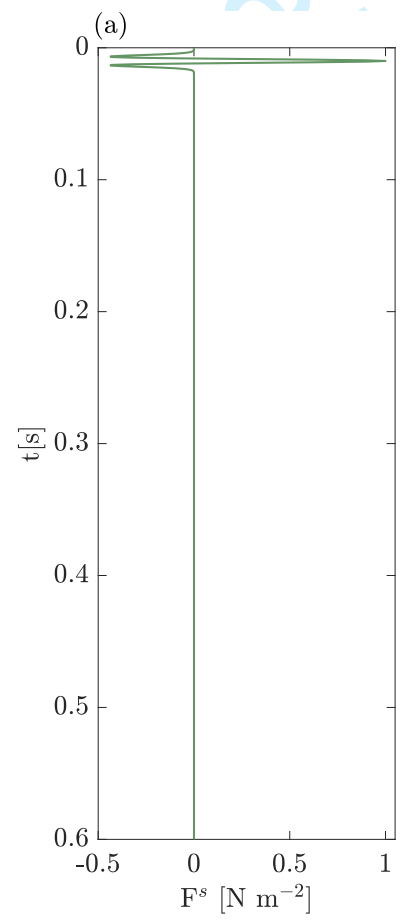

(b)

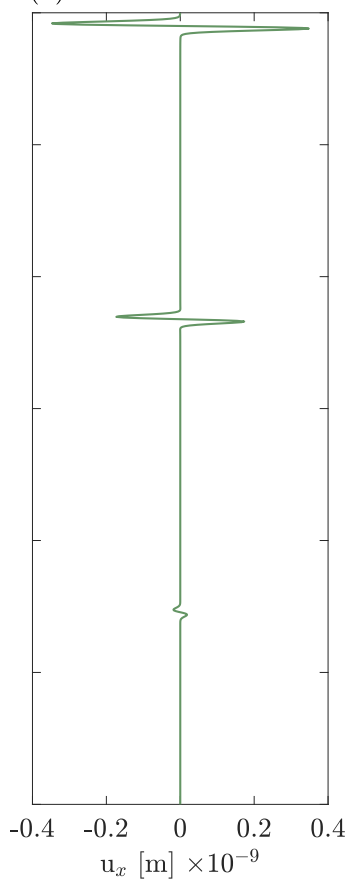

(c)

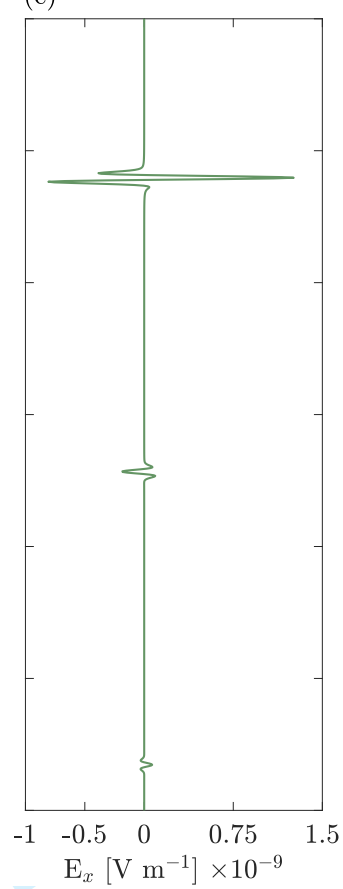

FIGURE 2 (a) Time signature of the shearing force $F^{s}(t)$ employed as source, (b) the solid displacement $u_{x}(t)$ and the electric field response $E_{x}(t)$, both computed at the surface (both (b) and (c) are the noiseless data with which the synthetic data are computed). 

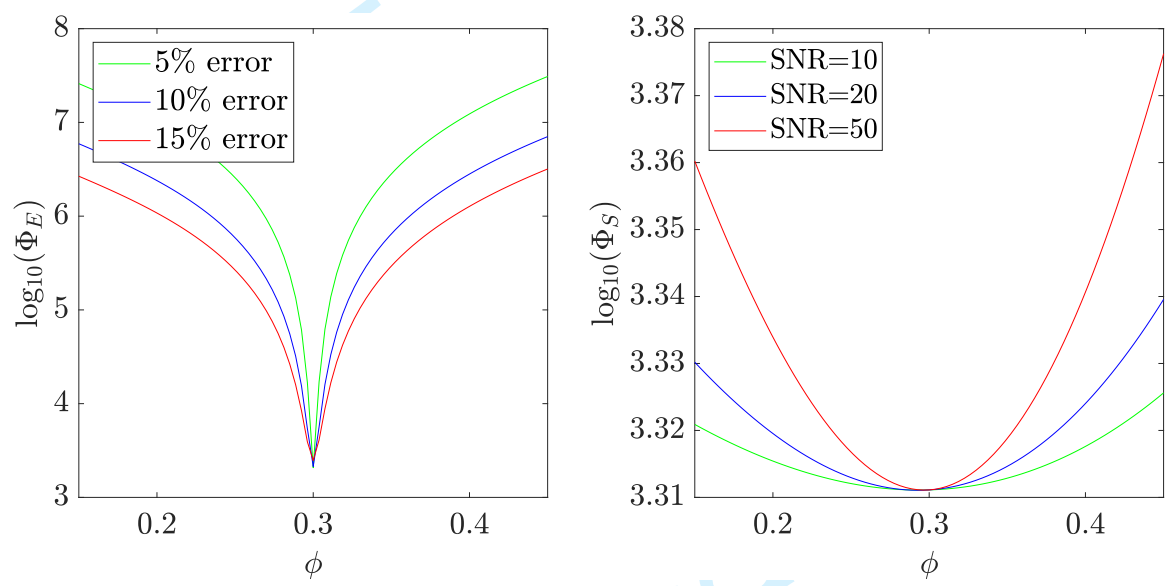

FIGURE 3 Misfits computed as a function of the porosity $\phi$. Fig. (a) displays the electric contribution to the total misfit, for three percentages of error in the electric data. Fig. (b) shows the seismic misfits due to three signal-to-noise ratios in the seismic data. Both the seismic and electric traces comprise all time samples, $N_{t}=2048$ 
1

2

3

4

5

6

7

8

9

10

11

12

13

14

15

16

17

18

19

20

21

22

23

24

25

26

27

28

29

30

31

32

33

34

35

36

37

38

39

40

41

42

43

44

45

46

47

48

49

50

51

52

53

54
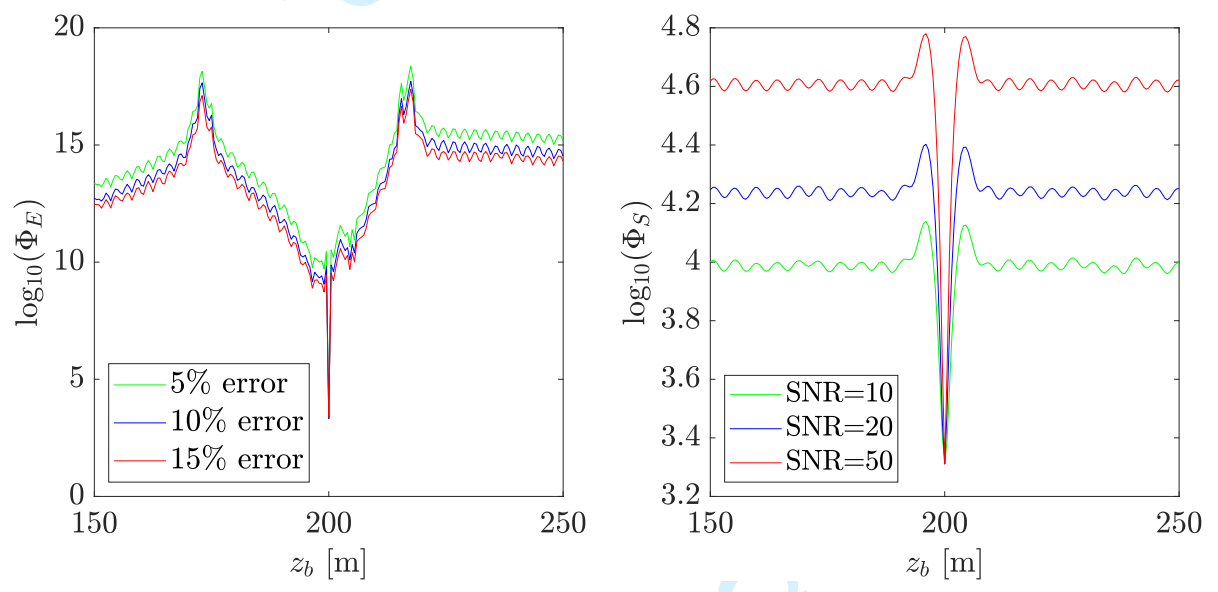

FIGURE 4 Misfits computed as a function of the depth of the top of the basement $z_{\mathrm{b}}$. Fig. (a) displays the electric contribution to the total misfit, for three percentages of error in the electric data. Fig. (b) shows the seismic misfits due to three signal-to-noise ratios in the seismic data. Both the seismic and electric traces comprise all time samples, $N_{t}=2048$ 
1

2

3

4

5

6

7

8

9

10

11

12

13

14

15

16

17

18

19

20

21

22

23

24

25

26

27

28

29

30

31

32

33

34

35

36

37

38

39

40

41

42

43

44

45

46

47

48

49

50

51

52

53

54

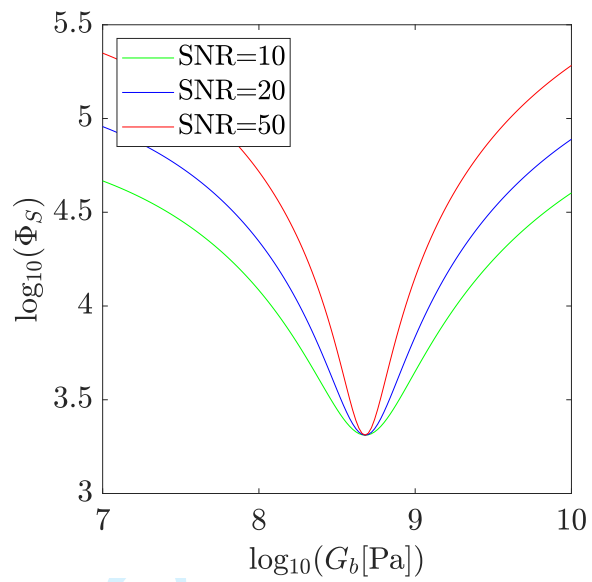

FIGURE 5 Misfits computed as a function of the basement rock matrix shear modulus $G_{\mathrm{b}}$. Fig. (a) displays the electric contribution to the total misfit, for three percentages of error in the electric data. Fig. (b) shows the seismic misfits due to three signal-to-noise ratios in the seismic data. Both the seismic and electric traces comprise all time samples, $N_{t}=2048$ 

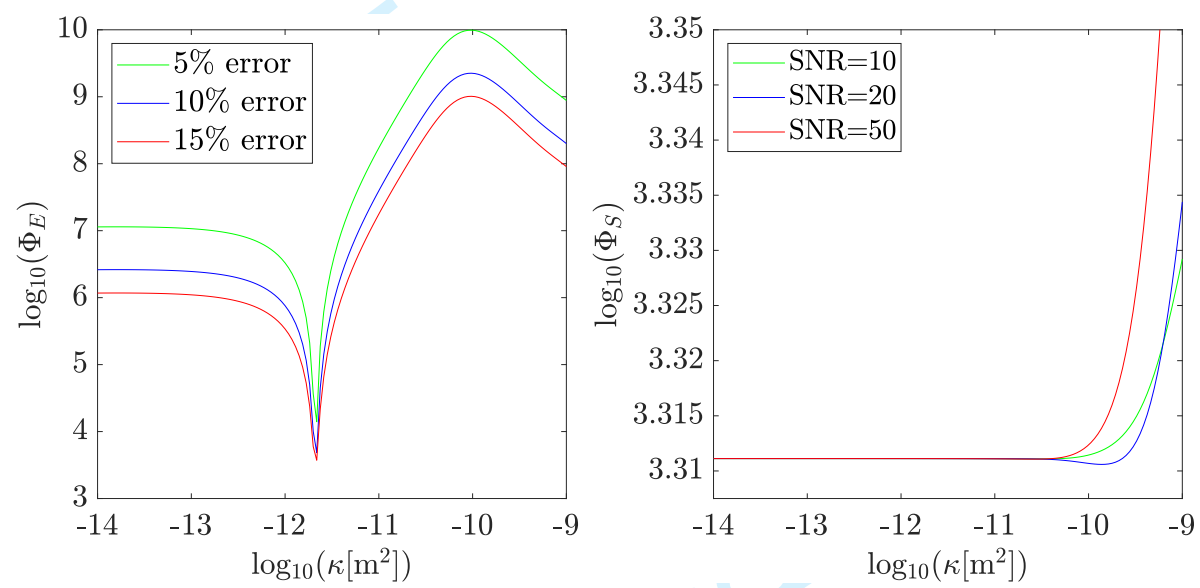

FIGURE 6 Misfits computed as a function of the permeability $k$. Fig. (a) displays the electric contribution to the total misfit, for three percentages of error in the electric data. Fig. (b) shows the seismic misfits due to three signal-to-noise ratios in the seismic data. Both the seismic and electric traces comprise all time samples, $N_{t}=2048$ 


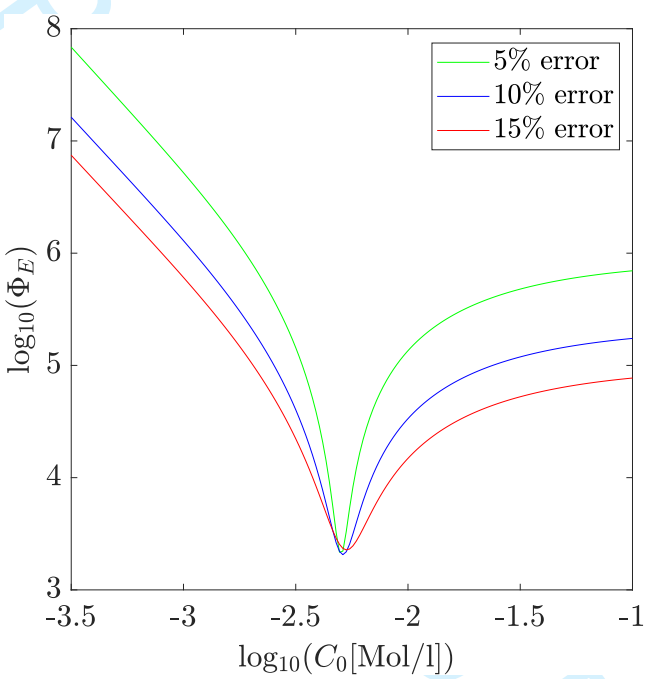

FIGURE 7 Electric misfit computed as a function of the water salinity, for three percentages of error in the electric data. We don't display the seismic misfits, because they are just constants, as expected. The electric trace comprises all time samples, $N_{t}=2048$. 

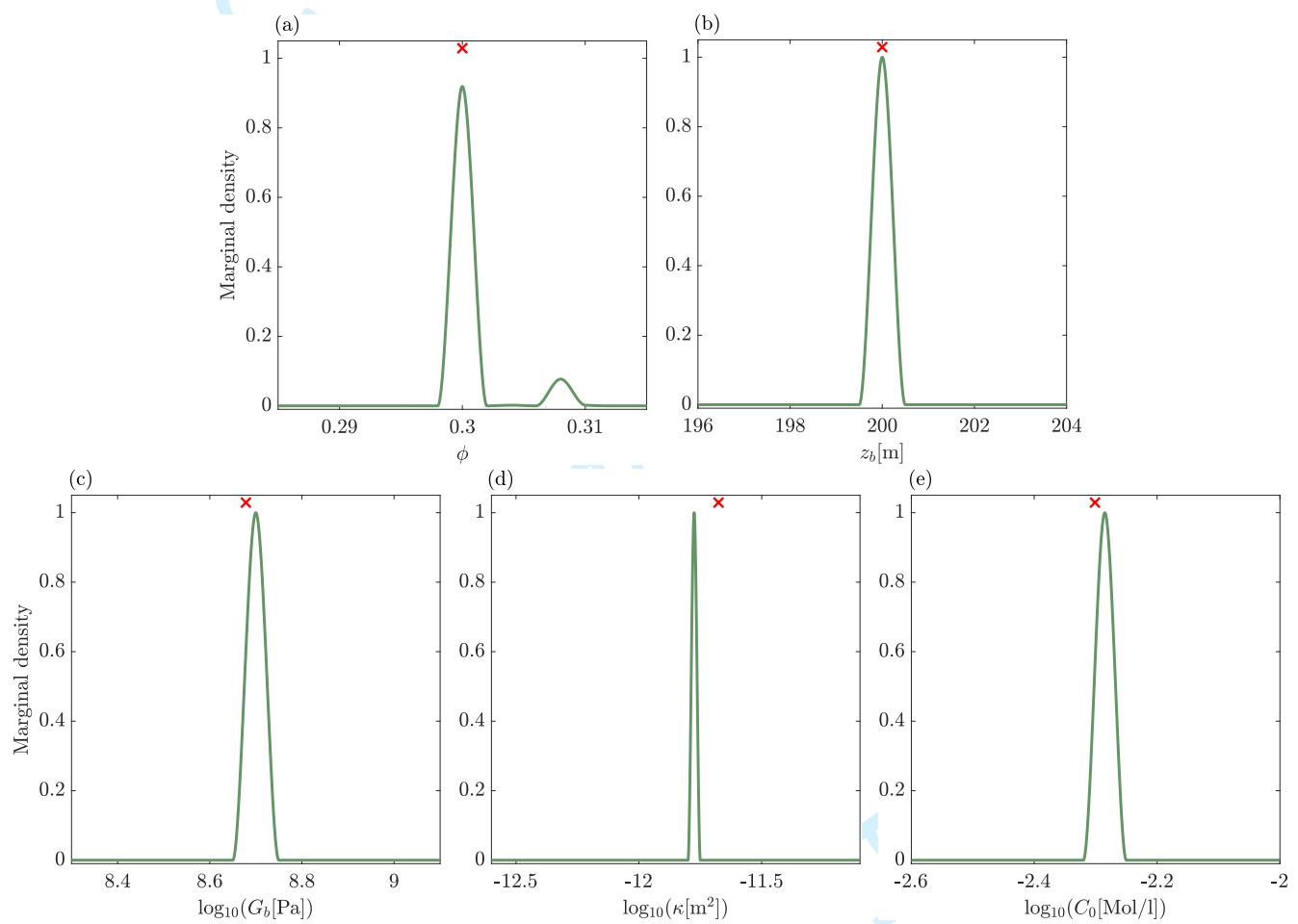

FIGURE 8 Marginal pdf, computed following the procedure described in Section 6.3 for (a) porosity, (b) glacier depth $z_{b}$, (c) $\log _{10}$ of basement shear modulus, $\log _{10}$ of saturating water salinity, $\log _{10}$ of permeability. Notice that the considered integration intervals are: [.28,.32] for $\phi$, [195,205] for $z_{b}$, [8.2,9.2] for $\log _{10}\left(G_{b}\right),[-2.6,-1.9]$ for $\log _{10}\left(C_{0}\right)$ and 39

40 

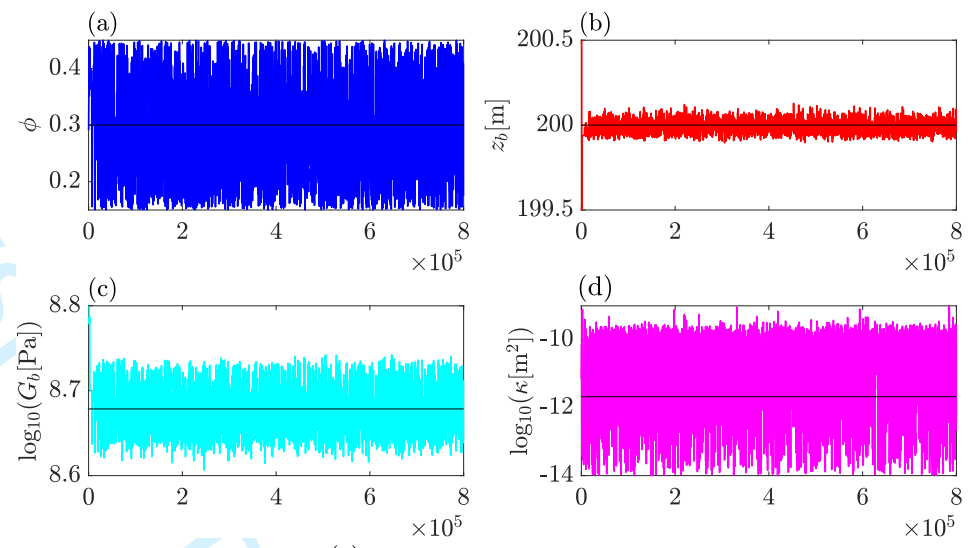

(e)

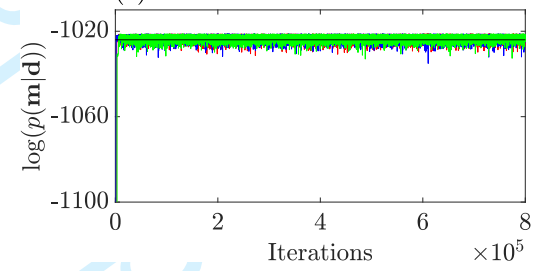

FIGURE 9 A single chain represent the search through the prior of the porosity (a), the depth (b), the bulk modulus (c) and the permeability (d) for the inversion of seismic data. Figure (e) shows the convergence of $\log (\mathrm{p}(\mathrm{m} \mid \mathrm{d})$ ) (for the three chains in color blue, red and green) to the expected value $-N_{t} / 2$, denoted by the solid horizontal black line. 
1

2

3

4

5

6

7

8

9

10

11

12

13

14

15

16

17

18

19

20

21

22

23

24

25

26

27

28

29

30

31

32

33

34

35

36

37

38

39

40

41

42

43

44

45

46

47

48

49

50

51

52

53

54
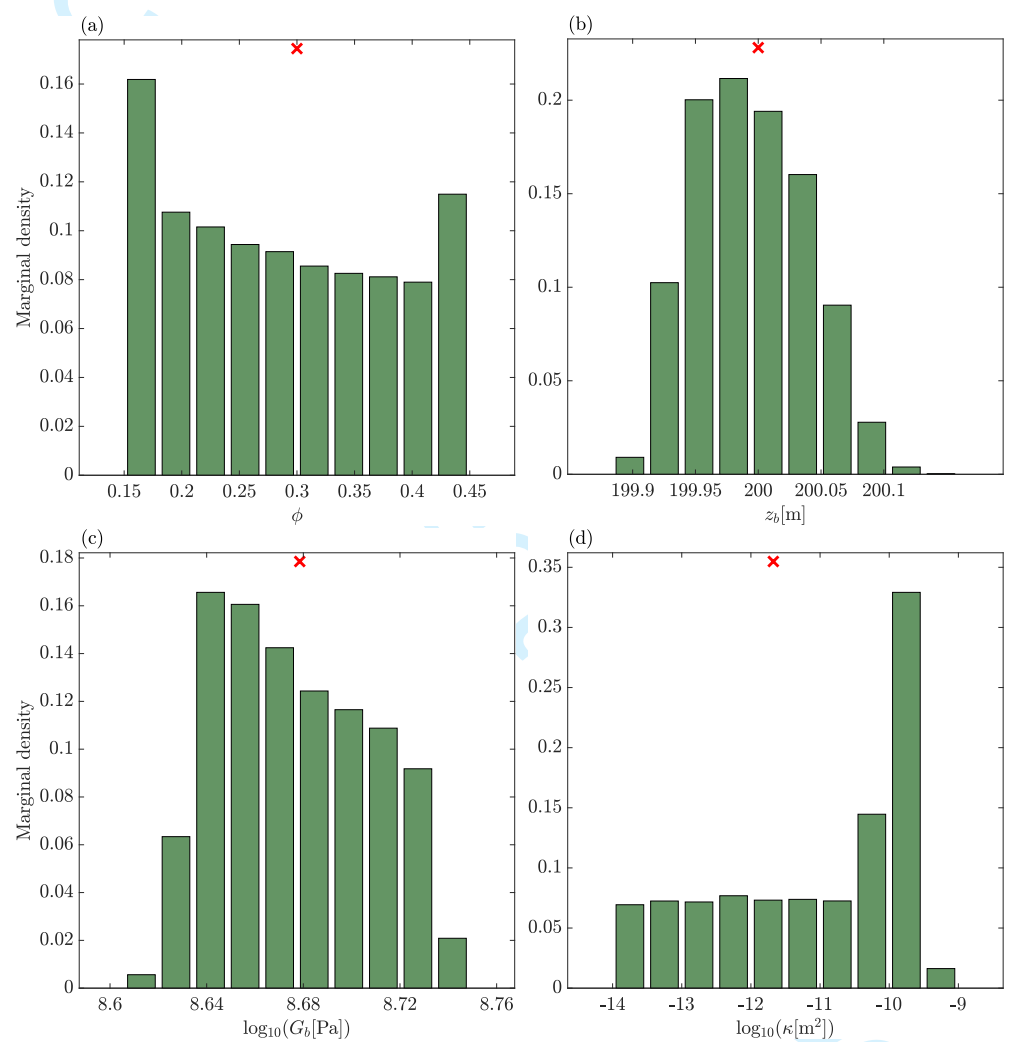

FIGURE 10 Histograms of the basement porosity (a), the depth (b), the bulk modulus (c) and the permeability (d) for the inversion of seismic data. The red cross indicates the true value for each parameter. 

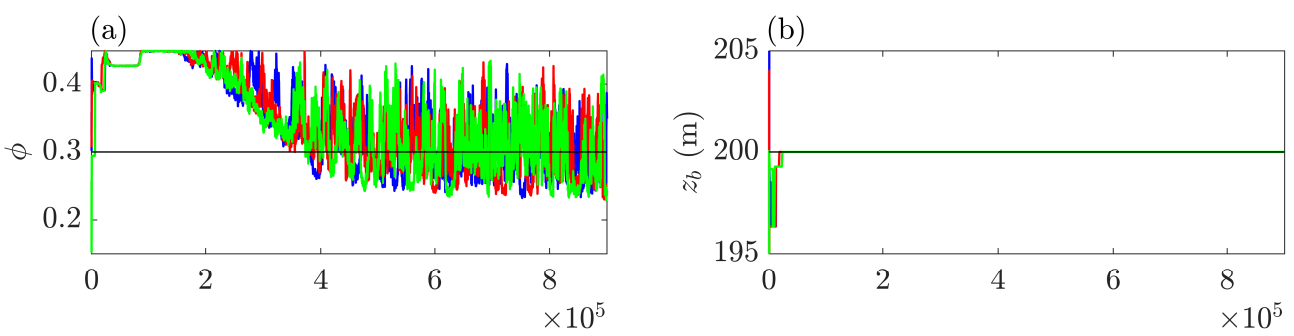

(c)

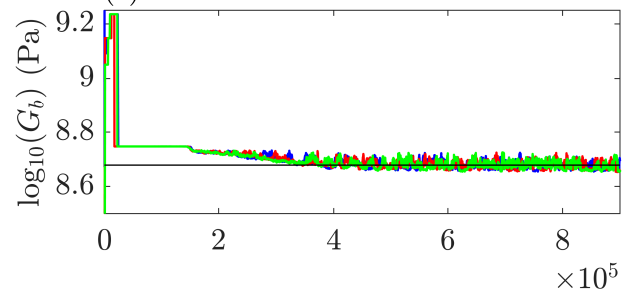

(e)

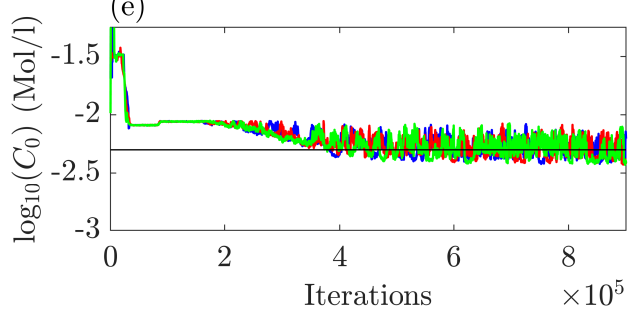

(f)

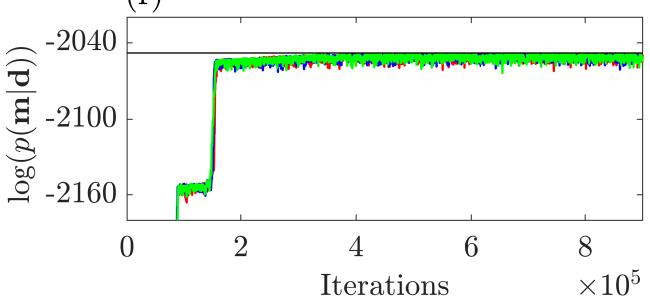

FIGURE 11 Figures (a) - (e) display the convergence of the chains for each parameter, for the joint inversion (each chain is represented by the colors blue, red and green). The chains move around the prior defined for each parameter. Figure ( $f$ ) shows the convergence of $\log (p(m \mid d))$ to the expected value $-N / 2$, denoted by the solid horizontal black line. 

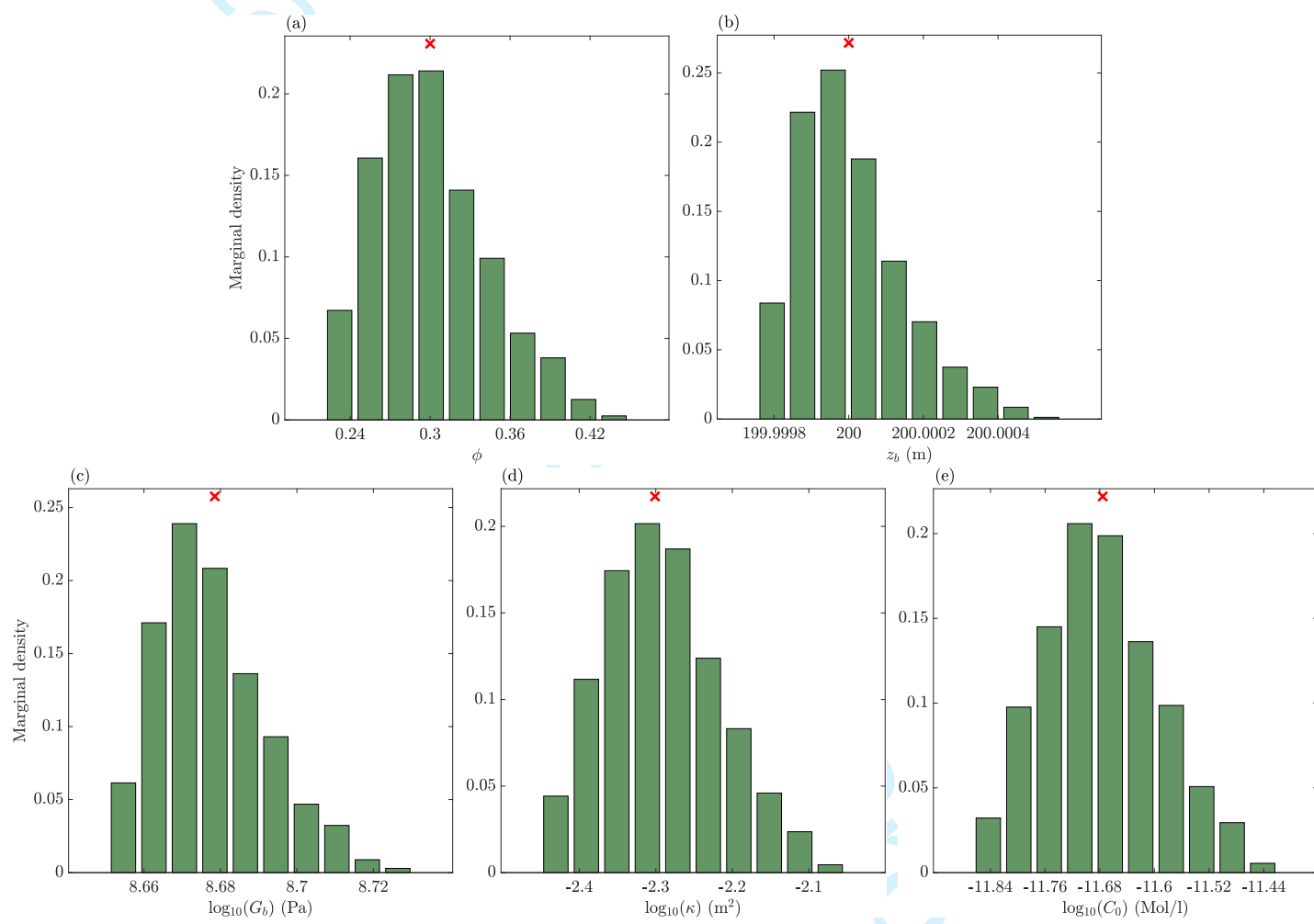

FIGURE 12 Histograms of the basement porosity (a), the depth (b), the bulk modulus (c), the permeability (d) and the salinity (e) for the inversion of electric data. The red cross indicates the true value for each parameter. 


\section{1}

2

3

4

5

6

7

8

9

10

11

12

13

14

15

16

17

18

19

20

21

22

23

24

25

26

27

28

29

30

31

32

33

34

35

36

37

38

39

40

41

42

43

44

45

46

47

48

49

50

51

52

53

54
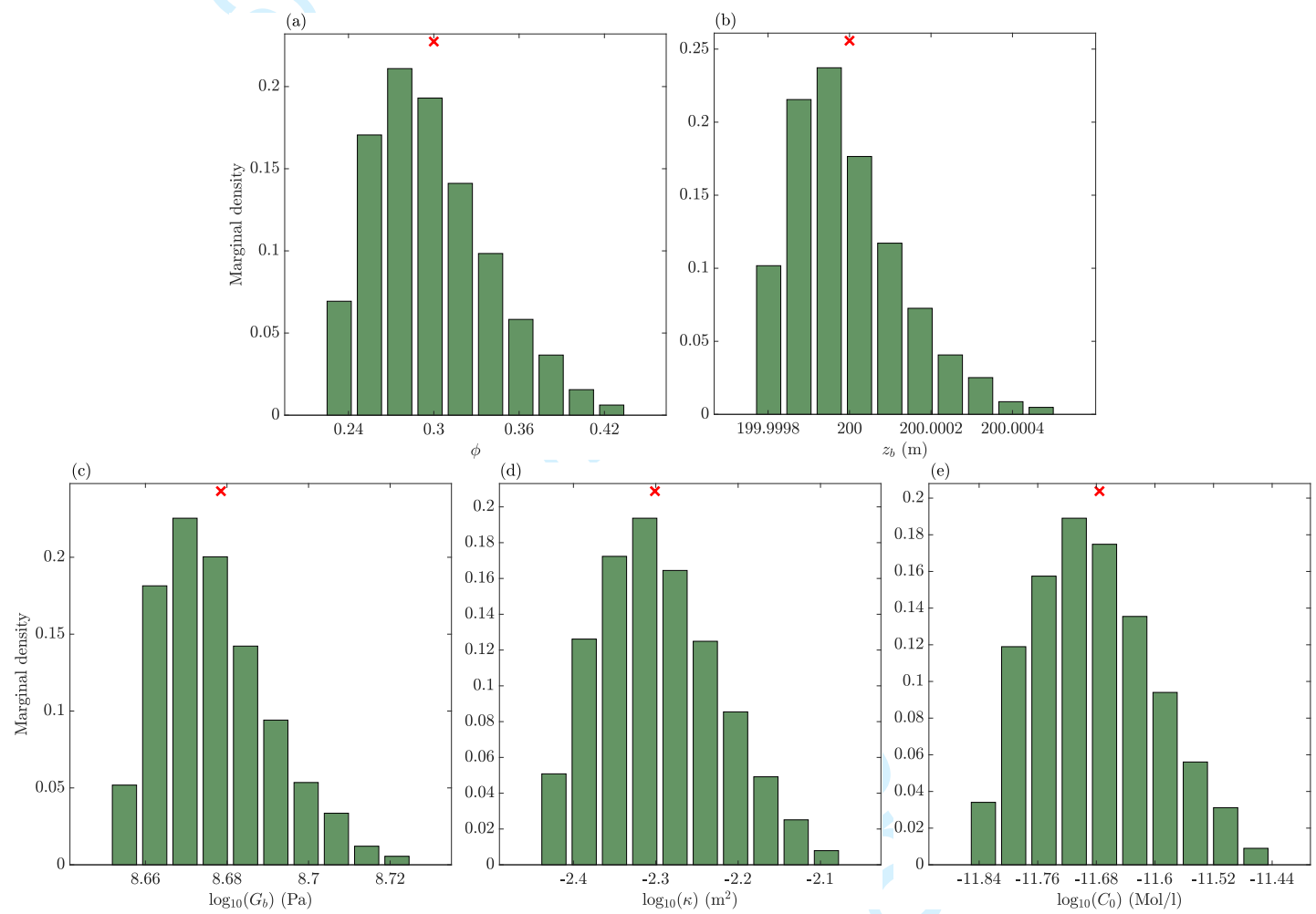

FIGURE 13 Histograms of the basement porosity (a), the depth (b), the bulk modulus (c), the permeability (d) and the salinity (e) for the inversion of seismoelectric data. The red cross indicates the true value for each parameter. 


\section{REFERENCES}

Aki, K. and Richards, P. G. (2002) Quantitative seismology (2nd Ed.). California: University Science Books.

Archie, G. E. (1942) The electrical resistivity log as an aid in determining some reservoir characteristics. Trans. Am. Inst. Min. Metall. Pet. Eng., 1, 54-62.

Bonnetier, E., Triki, F. and Xue, Q. (2019) An inverse problem for an electroseismic model describing the coupling phenomenon of electromagnetic and seismic waves. Inverse Problems, 35, 1-20.

Bordes, C. (2005) Etude expérimentale des phénomènes transitoires sismo-électromagnétiques: Mise en oeuvre au Laboratoire Souterrain à Bas Bruit de Rustrel. Ph.D. thesis, Université Joseph Fourier, Grenoble 1.

Bordes, C., Sénéchal, P., Barrière, J., Brito, D., Normandin, E. and Jougnot, D. (2015) Impact of water saturation on seismoelectric transfer functions: a laboratory study of coseismic phenomenon. Geophys. J. Int., 200, 1317-1335.

Brandt, S. (1989) Statistical and computational methods in data analysis. Elsevier.

Carcione, J., Seriani, G. and Gei, D. (2003) Acoustic and electromagnetic properties of soil saturated with salt water and NAPL. J. Appl. Geophys., 52, 177-191.

Chen, J., Hoversten, G. M., Vasco, D., Rubin, Y. and Hou, Z. (2007) A bayesian model for gas saturation estimation using marine seismic ava and csem data. Geophysics, 72, WA85-WA95.

Chen, J. and Yang, Y. (2013) Inverse problem of electro-seismic conversion. Inverse Problems, 29, 1-15.

Collins, J., Frank, S. and Metzler, A. (2016) Elastic parabolic equation and normal mode solutions for seismo-acoustic propagation in underwater environments with ice covers. J. Acoust. Soc. Am., 139, 2672.

Devi, M., Garambois, S., , Brito, D., Dietrich, M., Poydenot, V. and Bordes, C. (2018) A novel approach for seismoelectric measurements using multielectrode arrangements - II: laboratory measurements. Geophys. J. Int., 214, 1783-1799.

Dietrich, M., Devi, M., Garambois, S., , Brito, D. and Bordes, C. (2018) A novel approach for seismoelectric measurements using multielectrode arrangements - I: theory and numerical experiments. Geophys. J. Int., 215, 61-80.

Dupuy, B., Garambois, S. and Virieux, J. (2016) Estimation of rock physics properties from seismic attributes - Part 1: Strategy and sensitivity analysis. Geophysics, 80, M35-M53.

Fiorentino, E., Toussaint, R. and Jouniaux, L. (2017) Two-phase lattice boltzmann modelling of streaming potentials: influence of the gas-water interface on the electrokinetic coupling. Geophys. J. Int., 208, 1139-1156.

Fournier, C. (1989) Spontaneous potentials and resistivity surveys applied to hydrogeology in a volcanic area: Case history of the chaîne des puys (puy-de-dôme, france). Geophysical Prospecting, 37, 647-668.

French, H. K., Binley, A., Kharkhordin, I., Kulessa, B. and Krylov, S. S. (2006) Cold regions hydrogeophysics: Physical characterisation and monitoring. In Applied Hydrogeophysics, 195-232. Springer.

Gao, Y., Huang, F. and Hu, H. (2017a) Comparison of full and quasi-static seismoelectric analytically-based modeling. J. Geophys. Res.: Solid Earth, doi:10.1002/2017JB014251.

Gao, Y., Wang, D., Yao, C., , Guan, W., Hu, H., Zhang, W., Tong, P. and Yang, Q. (2019) Simulation of seismoelectric waves using finite-difference frequency-domain method: 2D SHTE mode. Geophys. J. Int., 216, 414-438.

Gao, Y., Wang, M., Hu, H. and Chen, X. (2017b) Seismoelectric responses to an explosive source in a fluid above a fluid-saturated porous medium. Journal of Geophysical Research: Solid Earth, 122, 7190-7218.

Garambois, S. and Dietrich, M. (2002) Full waveform numerical simulations of seismoelectromagnetic wave conversions in fluid-saturated stratified porous media. J. Geophys. Res., 107, ESE 5-1. 
Gelman, A., Rubin, D. B. et al. (1992) Inference from iterative simulation using multiple sequences. Statistical science, 7, 457472.

Gilks, W. R., Richardson, S. and Spiegelhalter, D. (1995) Markov chain Monte Carlo in practice. Chapman and Hall/CRC.

Grobbe, N. and Slob, E. (2016) Seismo-electromagnetic thin-bed responses: Natural signal enhancements? J. Geophys. Res.

Guan, W., Hu, H. and Wang, Z. (2013) Permeability inversion from low-frequency seismoelectric logs in fluid-saturated porous formations. Geophysical Prospecting, 61, 120-133.

Guan, W., Shi, P. and Hu, H. (2017) Contributions of poroelastic-wave potentials to seismo- electromagnetic wavefields and validity of the quasi-static calculation: A view from a borehole model. Geophys. J. Int.

Gueguen, Y. and Palciauskas, V. (1994) Introduction to the Physics of Rocks. Princeton: Princeton University Press.

Haartsen, M. W. and Pride, S. (1997) Electroseismic waves from point sources in layered media. J. Geophys. Res., 102, 24,74524,769 .

Hahn, T. (2005) Cuba-a library for multidimensional numerical integration. Computer Physics Communications, 168, 78-95.

Haines, S. H. and Pride, S. R. (2006) Seismoelectric numerical modeling on a grid. Geophysics, 71, 57-65.

Haines, S. S., Guitton, A. and Biondi, B. (2007a) Seismoelectric data processing for surface surveys of shallow targets. Geophysics, 72, G1-G8.

Haines, S. S., Pride, S. R., Klemperer, S. L. and Biondi, B. (2007b) Seismoelectric imaging of shallow targets. Geophysics, 72 , G9-G20.

Hassan, A. E., Bekhit, H. M. and Chapman, J. B. (2009) Using markov chain monte carlo to quantify parameter uncertainty and its effect on predictions of a groundwater flow model. Environmental Modelling \& Software, 24, 749-763.

Holzhauer, J., Brito, D., Bordes, C., Brun, Y. and Guatarbes, B. (2016) Experimental quantification of the seismoelectric transfer functions and its dependence on conductivity and saturation in loose sand. Geophysical Prospecting.

Hu, H. and Gao, Y. (2011) Electromagnetic field generated by a finite fault due to electrokinetic effect. J. Geophys. Res., 116, 1132-1143,.

Hu, H., Guan, W. and Harris, J. (2007) Theoretical simulation of electroacoustic borehole logging in a fluid-saturated porous formation. J. Acoust. Soc. Am., 122, 135-145.

$\mathrm{Hu}, \mathrm{H}$. and Liu, J. (2002) Simulation of the converted electric field during acoustoelectric logging. 72nd SEG Annual International Meeting, Expanded Abstracts,21, 348-351.

Hu, H., Wang, J. and Guan, W. (2015) Experimental measurements of seismoelectric signals in borehole models. Geophysical Journal International, 203, 1937-1945.

Jardani, A. and Revil, A. (2015) Seismoelectric couplings in a poroelastic material containing two immiscible fluid phases. Geophys. J. Int., 202, 850-870.

Jardani, A., Revil, A., Slob, E. and Söllner, W. (2010) Stochastic joint inversion of 2d seismic and seismoelectric signals in linear poroelastic materials: A numerical investigation. geophysics, 75, N19-N31.

Jeffreys, H. (1998) The theory of probability. OUP Oxford.

Johnson, D. L., Koplik, J. and Dashen, R. (1987) Theory of dynamic permeability in fluid saturated porous media. J. Fluid. Mech., 176, 379-402. 
Jougnot, D., Rubino, J., Carbajal, M. R., Linde, N. and Holliger, K. (2013) Seismoelectric effects due to mesoscopic heterogeneities. Geophys. Res. Lett., 40, 2033-2037.

Jouniaux, L. and Zyserman, F. (2016) A review on electrokinetically induced seismo-electrics, electro-seismics, and seismomagnetics for Earth sciences. Solid Earth, 7, 249-284.

Krawczyk, C., Polom, U. and Beilecke, T. (2013) Shear-wave reflection seismics as a valuable tool for near-surface urban applications. The Leading Edge, 32, 253-263.

Kröger, B., Yaramanci, U. and Kemna, A. (2014) Numerical analysis of seismoelectric wave propagation in spatially confined geological units. Geophysical Prospecting, 62, 133-147.

Kulessa, B., Hubbard, B. and Brown, G. (2006a) Time-lapse imaging of subglacial drainage conditions using 3-D inversion of subglacial electrical resistivity. J. Glaciol., 42, 49-57.

Kulessa, B., Hubbard, B., Brown, G. and Becker, J. (2003) Earth tide forcing of glacier drainage. Geophys. Res. Lett., 30.

Kulessa, B. and Murray, T. (2003) Slug-test derived differences in bed hydraulic properties between a surge-type and a nonsurge type Svalbard glacier. Ann. Glaciol., 36, 103-109.

Kulessa, B., Murray, T. and Rippin, D. (2006b) Active seismoelectric exploration of glaciers. Geophys. Res. Lett., 33, L07503.

Liu, Z., Yuan, L., Zhang, X., Liu, Z. and Wu, H. (2008) A laboratory seismoelectric measurement for the permafrost model with a frozen-unfrozen interface. Geophys. Res. Lett., 35, L21404.

Løseth, L., Pedersen, H., Ursin, B., Amundsen, L. and Ellingsrud, S. (2006) Low-frequency electromagnetic fields in applied geophysics: Waves or diffusion? Geophysics, 71, W29-W40.

Mahardika, H. (2013) Coupled Hydromechanical and Electromagnetic Responses in Unsaturated Porous Media: Theory, Observation, and Numerical Simulations. Ph.D. thesis, Colorado School of Mines.

Mahardika, H., Revil, A. and Jardani, A. (2012) Waveform joint inversion of seismograms and electrograms for moment tensor characterization of fracking events. Geophysics, 77, ID23-ID39.

Mavko, G., Mukerji, T. and Dvorkin, J. (2009) The rock physics handbook: Tools for seismic analysis of porous media. The Pitt Building, Trumpington Street, Cambridge CB2 IRP, United Kingdom: Cambridge University Press.

Monachesi, L., Zyserman, F. and Jouniaux, L. (2018a) An analytical solution to assess the SH seismoelectric response of the vadose zone. Geophysical Journal International, 213, 1999-2019.

- (2018b) SH Seismoelectric Response of a Glacier: An Analytic Study. J. Geophys. Res.: Earth Surface, 123, 2135-2156.

Mosegaard, K. and Tarantola, A. (1995) Monte carlo sampling of solutions to inverse problems. Journal of Geophysical Research: Solid Earth, 100, 12431-12447.

Munch, F. and Zyserman, F. (2016) Detection of Non-Aqueous Phase Liquids Contamination by SH-TE Seismoelectrics: a Computational Feasibility Study. Journal of Applied Geophysics.

Peng, R., Di, B., Wei, J., Ding, P., Zhao, J., Pan, X. and Liu, Z. (2017) Experimental study of the seismoelectric interface response in wedge and cavity models. Geophys. J. Int., 210, 1703-1720.

Petrenko, V. and Whitworth, R. (1999) Physics of Ice. Clarendon Press.

Press, W., Teukolsky, S., Vetterling, W. and Flannery, B. (2007) Numerical Recipes, The art of scientific computing. Cambridge University Press, 3rd edn.

Pride, S. (1994) Governing equations for the coupled electromagnetics and acoustics of porous media. Phys. Rev. B: Condens. Matter, 50, 15678-15695. 
Pride, S. R. (2005) Relationships between seismic and hydrological properties. In Hydrogeophysics, chap. 9, 253-291. Dordrecht, The Netherlands: Springer.

Rapetti, F. and Rousseaux, G. (2014) On quasi-static models hidden in Maxwell's equations. Applied Numerical Mathematics, 79, 92-106.

Ren, H., Huang, Q. and Chen, X. (2016) Existence of evancescent electromagnetic waves resulting from seismoelectric conversion at a solid-porous interface. Geophys. J. Int., 204, 147-166.

Revil, A., Jardani, A., Sava, P. and Haas, A. (2015) The Seismoelectric Method: Theory and Application. Wiley Blackwell.

Revil, A. and Mahardika, H. (2013) Coupled hydromechanical and electromagnetic disturbances in unsaturated porous materials. Water Resources Research, 49, 744-766.

Robinson, E. A. and Treitel, S. (2000) Geophysical signal analysis. Society of Exploration Geophysicists.

Rosas-Carbajal, M., Linde, N., Kalscheuer, T. and Vrugt, J. A. (2014) Two-dimensional probabilistic inversion of plane-wave electromagnetic d ata: Methodology, model constraints and joint inversion with electrical resist ivity data. Geophys. J. Int., 196, 1508-1524.

Rosas-Carbajal, M., Linde, N., Peacock, J., Zyserman, F., Kalscheuer, T. and Thiel, S. (2015) Probabilistic three-dimensional timelapse inversion of magnetotelluric data to infer mass transfer in a geothermal system. Geophys. J. Int., 203, 1946-1960.

Sambdridge, M. and Mosegaard, K. (2002) Monte carlo methods in geophysical inverse problems. Reviews of Geophysics, 40, $1-29$.

Sambridge, M. and Drijkoningen, G. (1992) Genetic algorithms in seismic waveform inversion. Geophysical Journal International, $109,323-342$.

Santos, J., Ravazzoli, C., Gauzellino, P. and Carcione, J. (2005) Numerical simulation of ultrasonic waves in reservoir rocks with patchy saturation and fractal petrophysicas properties. Computational Geosciences, 9, 1-27.

Santos, J., Ravazzoli, C., Gauzellino, P., Carcione, J. and Cavallini, F. (2004) Simulation of waves in poro-viscoelastic rocks saturated by immiscible fluids. numerical evidence of a second slow wave. J. Comput. Acoust., 12, 1-21.

Santos, J. E. (2009) Finite element approximation of coupled seismic and electromagnetic waves in fluid-saturated poroviscoelastic media. Numerical Methods for Partial Differential Equations, 27, 351-386.

Schön, J. (1996) Physical properties of rocks - fundamentals and principles of petrophysics, vol. 18. Handbook of Geophysical Exploration, Seismic exploration: Elsevier Science Ltd.

Sen, M. K. and Stoffa, P. L. (1992) Rapid sampling of model space using genetic algorithms: examples from seismic waveform inversion. Geophysical Journal International, 108, 281-292.

Siegert, M. J., Kulessa, B., Bougamont, M., Christoffersen, P., Key, K., Andersen, K. R., Booth, A. D. and Smith, A. M. (2018) Antarctic subglacial groundwater: a concept paper on its measurement and potential influence on ice flow. Geological Society, London, Special Publications, 461, 197-213.

Tarantola, A. (2005) Inverse problem theory and methods for model parameter estimation, vol. 89. siam.

Tarantola, A. and Valette, B. (1982) Inverse problems= quest for information. J. geophys, 50, 150-170.

Ter Braak, C. J. (2006) A markov chain monte carlo version of the genetic algorithm differential evolution: easy bayesian computing for real parameter spaces. Statistics and Computing, 16, 239-249.

Vrugt, J., ter Braak, C., Diks, C., Robinson, B., Hyman, J. and Higdon, D. (2009) Accelerating Markov chain Monte Carlo simulation by Differential Evolution with Self-Adaptive Randomized Subpace Sampling. International Journal of Nonlinear Sciences and Numerical Simulation, 10, 273-290. 
Warden, S., Garambois, S., Jouniaux, L., Brito, D., Sailhac, P. and Bordes, C. (2013) Seismoelectric wave propagation numerical modeling in partially saturated materials. Geophys. J. Int., 194, 1498-1513.

Warden, S., Garambois, S., Sailhac, P., Jouniaux, L. and Bano, M. (2012) Curvelet-based seismoelectric data processing. Geophys. J. Int., 190, 1533-1550.

Zyserman, F., Gauzellino, P. and Santos, J. (2012) Numerical evidence of gas hydrate detection by means of electroseismics. J. Applied Geophysics, 86, 98-108.

Zyserman, F., Jouniaux, L., Warden, S. and Garambois, S. (2015) Borehole seismoelectric logging using a shear-wave source:Possible application to $\mathrm{CO}_{2}$ disposal? International Journal of Greenhouse Gas Control, 33, 82-102.

Zyserman, F., Monachesi, L. and Jouniaux, L. (2017a) Dependence of shear wave seismoelectrics on soil textures: a numerical study in the vadose zone. Geophys. J. Int., 208, 918-935.

- (2017b) Reply to "Comment on "Dependence of shear wave seismoelectrics on soil textures: a numerical study in the vadose zone by F.I. Zyserman, L.B. Monachesi and L. Jouniaux" by Revil, A. Geophys. J. Int., 210, 1652-1658. 


\section{APPENDIX: FinAl SOLUTIONS FOR $u_{x}, E_{x}$ AND $H_{y}$.}

The final solution for the solid displacement $u_{x}$ is given by (Monachesi et al., 2018b):

$$
u_{x}(z)=\left\{\begin{array}{l}
-\frac{F^{s}}{i i_{\text {ice }} G_{\text {ice }}} e^{i \lambda_{\text {ice }} z}+\sum_{n=1}^{\infty} U_{x}^{R, z_{\mathrm{b}},(n)} e^{-i \lambda_{\text {ice }}\left(z-z_{\mathrm{b}}\right)}+\sum_{n=1}^{\infty} U_{x}^{R, 0,(n)} e^{i \lambda_{\text {ice }} z}, \quad 0 \leq z \leq z_{\mathrm{b}}, \\
\sum_{n=1}^{\infty} U_{s, x}^{(n)} e^{i \lambda_{\mathrm{b}}\left(z-z_{\mathrm{b}}\right)}, \quad z \geq z_{\mathrm{b}},
\end{array}\right.
$$

where:

$$
\begin{gathered}
U_{x}^{R, z_{\mathrm{b}},(n)}=\frac{i F^{s}\left(\lambda_{\text {ice }} G_{\text {ice }}-\lambda_{\mathrm{b}} G_{\mathrm{b}}\right)^{n} e^{i(2 n-1) \lambda_{\mathrm{b}} z_{\mathrm{b}}}}{\lambda_{\text {ice }} G_{\text {ice }}\left(\lambda_{\text {ice }} G_{\text {ice }}+\lambda_{\mathrm{b}} G_{\mathrm{b}}\right)^{n}}, \\
U_{x}^{R, 0,(n)}=\frac{i F^{s}\left(\lambda_{\text {ice }} G_{\text {ice }}-\lambda_{\mathrm{b}} G_{\mathrm{b}}\right)^{n} e^{i 2 n \lambda_{\text {ice }} z_{\mathrm{b}}}}{\lambda_{\text {ice }} G_{\text {ice }}\left(\lambda_{\text {ice }} G_{\text {ice }}+\lambda_{\mathrm{b}} G_{\mathrm{b}}\right)^{n}} . \\
U_{s, x}^{(n)}=\frac{2 i F^{s}\left(\lambda_{\text {ice }} G_{\text {ice }}-\lambda_{\mathrm{b}} G_{\mathrm{b}}\right)^{(n-1)} e^{i(2 n-1) \lambda_{\text {ice }} z_{\mathrm{b}}}}{\left(\lambda_{\text {ice }} G_{\text {ice }}+\lambda_{\mathrm{b}} G_{\mathrm{b}}\right)^{n}},
\end{gathered}
$$

In these equations, $\lambda_{\text {ice }}$ and $\lambda_{\mathrm{b}}$ are the S-wave seismic wave numbers of the glacier and basement, respectively, and are given by:

$$
\lambda_{\text {ice }}=\omega \sqrt{\frac{\rho_{\text {ice }}}{G_{\text {ice }}}}, \quad \lambda_{\mathrm{b}}=\omega \sqrt{\frac{1}{G_{\mathrm{b}}}\left(\rho_{\mathrm{b}}-\frac{\rho_{\mathrm{w}}^{2}}{g_{0}-i \eta_{\mathrm{w}} /(\kappa \omega)}\right)},
$$

The summation appearing in eq.(31) is made over the $n$-th reflection/transmission of the seismic wave at $z=z_{\mathrm{b}}$. The electric and magnetic fields are given by:

$$
\begin{aligned}
& E_{x}(z)=\left\{\begin{array}{l}
A_{\text {ice }} e^{-i k_{\text {ice }} z}+B_{\text {ice }} e^{i k_{\text {ice }} z \quad} \quad 0 \leq z \leq z_{\mathrm{b}}, \\
A_{\mathrm{b}} e^{-i k_{\mathrm{b}} z}+B_{\mathrm{b}} e^{i k_{\mathrm{b}} z}-\frac{k_{\mathrm{b}}^{2} e^{i \lambda_{\mathrm{b}}\left(z-z_{\mathrm{b}}\right)}}{\left(k_{\mathrm{b}}^{2}-\lambda_{\mathrm{b}}^{2}\right) \sigma_{\mathrm{b}}} \sum_{n=1}^{\infty} J_{v}^{(n)} \quad z \geq z_{\mathrm{b}} .
\end{array}\right. \\
& H_{y}(z)=\left\{\begin{array}{l}
\frac{k_{\text {ice }}}{\omega \mu_{0}} A_{\text {ice }} e^{-i k_{\text {ice }} z}-\frac{k_{\text {ice }}}{\omega \mu_{0}} B_{\text {ice }} e^{i k_{\text {ice }} z \quad} \quad 0 \leq z \leq z_{\mathrm{b}}, \\
\frac{k_{\mathrm{b}}}{\omega \mu_{0}} A_{\mathrm{b}} e^{-i k_{\mathrm{b}} z}-\frac{k_{\mathrm{b}}}{\omega \mu_{0}} B_{\mathrm{b}} e^{i k_{\mathrm{b}} z}-\frac{i \lambda_{\mathrm{b}} e^{i \lambda_{\mathrm{b}}\left(z-z_{\mathrm{b}}\right)}}{k_{\mathrm{b}}^{2}-\lambda_{\mathrm{b}}^{2}} \sum_{n=1}^{\infty} J_{v}^{(n)} \quad z \geq z_{\mathrm{b}} .
\end{array}\right.
\end{aligned}
$$

The coefficients $k_{\text {ice }}=\sqrt{-i \omega \mu_{0} \sigma_{\text {ice }}}$ and $k_{\mathrm{b}}=\sqrt{-i \omega \mu_{0} \sigma_{\mathrm{b}}}$ are the electromagnetic wave numbers of ice and basement, respectively. $J_{v}^{(n)}$ is the amplitude of the $n$-th current density originated at the $n$-th transmission of the seismic shear wave at $z=z_{\mathrm{b}}$, and is given by:

$$
J_{v}^{(n)}=\frac{\omega \frac{\eta_{\mathrm{w}}}{\kappa} L_{0} \rho_{w}}{\left(g_{0}-\frac{i \eta_{\mathrm{w}}}{\omega \kappa}\right)} \frac{2 F^{s}\left(\lambda_{\text {ice }} G_{\text {ice }}-\lambda_{\mathrm{b}} G_{\mathrm{b}}\right)^{(n-1)} e^{i(2 n-1) \lambda_{\text {ice }} z_{\mathrm{b}}}}{\left(\lambda_{\text {ice }} G_{\text {ice }}+\lambda_{\mathrm{b}} G_{\mathrm{b}}\right)^{n}}
$$


Finally, the coefficients $A_{\text {ice }}, B_{\text {ice }}, A_{\mathrm{b}}$, and $B_{\mathrm{b}}$ are complex constants which are determined by imposing proper conditions for the fields at the contact between both mediums and at the boundaries of the system. These constants are given by:

$$
\begin{gathered}
A_{\text {ice }}=B_{\text {ice }}=\frac{k_{\mathrm{b}}^{2}\left(\lambda_{\mathrm{b}}-k_{\mathrm{b}}\right)^{-1}}{2\left[k_{\text {ice }} \sinh \left(i k_{\text {ice }} z_{\mathrm{b}}\right)+k_{\mathrm{b}} \cosh \left(i k_{\text {ice }} z_{\mathrm{b}}\right)\right] \sigma_{\mathrm{b}}} \sum_{n=1}^{\infty} J_{v}^{(n)}, \\
A_{\mathrm{b}}=\frac{k_{\mathrm{b}}^{2}\left[k_{\text {ice }} \sinh \left(i k_{\text {ice }} z_{\mathrm{b}}\right)-\lambda_{\mathrm{b}} \cosh \left(i k_{\text {ice }} z_{\mathrm{b}}\right)\right] e^{i k_{\mathrm{b}} z_{\mathrm{b}}}}{\left(k_{2}^{2}-\lambda_{2}^{2}\right)\left[k_{\text {ice }} \sinh \left(i k_{\text {ice }} z_{\mathrm{b}}\right)+k_{\mathrm{b}} \cosh \left(i k_{\text {ice }} z_{\mathrm{b}}\right)\right] \sigma_{\mathrm{b}}} \sum_{n=1}^{\infty} J_{v}^{(n)}, \\
B_{\mathrm{b}}=0 .
\end{gathered}
$$

Once the fields $u_{x}(z, \omega), E_{x}(z, \omega)$ and $H_{y}(z, \omega)$ are computed for a given depth $z$, the time variation of these fields is obtained by the inverse Fourier transform. 